\title{
Model Builder for Item Factor Analysis with OpenMx
}

\author{
by Joshua N. Pritikin and Karen M. Schmidt
}

\begin{abstract}
We introduce a shiny web application to facilitate the construction of Item Factor Analysis (a.k.a. Item Response Theory) models using the OpenMx package. The web application assists with importing data, outcome recoding, and model specification. However, the app does not conduct any analysis but, rather, generates an analysis script. Generated Rmarkdown output serves dual purposes: to analyze a data set and demonstrate good programming practices. The app can be used as a teaching tool or as a starting point for custom analysis scripts.
\end{abstract}

\section{An overview of OpenMx}

OpenMx, a modular package originally designed for structural equation modeling (Neale et al., in press), recently gained the ability to handle Item Factor Analysis (a.k.a. Item Response Theory, Modern Test Theory) models (Pritikin et al., 2015). Although a goal of OpenMx is to cater to the statistical power user and facilitate analyses that are difficult to conduct in other software, the development team is always on the lookout for ways to ease the learning curve for novice users as well. Here we introduce a new shiny (RStudio and Inc., 2014) web application to generate OpenMx code in Rmarkdown format (Allaire et al., 2014). We believe this code generator substantially lowers the barrier to entry for novice users of Item Factor Analysis (IFA) and encourages a culture of literate programming (Knuth, 1984) and reproducible science (Peng, 2011; Nosek et al., 2015). The generated code can be customized at many levels. This flexibility enables the production of custom analyses and reports as users grow more sophisticated in their modeling expectations.

\section{The statistical model}

Item analysis is concerned with items that are scored correct/incorrect or on an ordinal scale. Many psychological surveys use an ordinal scale. For example, participants may be asked to respond to an item like, "I feel I am in charge of the situation in which I live." on a 5-point Likert scale from agree to disagree. Whether dichotomous or ordinal, the conditional likelihood of response $x_{i j}$ to item $j$ from person $i$ with item parameters $\xi_{j}$ and latent ability (a.k.a. latent trait) $\theta_{i}$ is

$$
L\left(x_{i} \mid \xi, \theta_{i}\right)=\prod_{j} \operatorname{Pr}\left(\text { pick }=x_{i j} \mid \xi_{j}, \theta_{i}\right) .
$$

One implication of Equation 1 is that items are assumed to be conditionally independent given the latent ability $\theta_{i}$. That is, the outcome of one item does not have any influence on another item after controlling for $\xi$ and $\theta_{i}$. The unconditional likelihood is obtained by integrating over the latent distribution $\theta_{i}$,

$$
L\left(x_{i} \mid \xi\right)=\int L\left(x_{i} \mid \xi, \theta_{i}\right) L\left(\theta_{i}\right) \mathrm{d} \theta_{i}
$$

With an assumption that examinees are independently and identically distributed, we can sum the individual log likelihoods,

$$
\mathcal{L}=\sum_{i} \log L\left(x_{i} \mid \xi\right)
$$

Optimization consists of finding the $\xi$ that maximizes this function. OpenMx presently offers only one choice for optimization, an Expectation-Maximization algorithm using equal interval quadrature to evaluate the integral in Equation 2 (Bock and Aitkin, 1981). In the future, we plan to add complementary algorithms such as Metropolis-Hastings Robbins-Monro, that is more efficient at optimizing certain problems (Cai, 2010b).

Several models are readily available to plug in as the response probability function $\operatorname{Pr}(\mathrm{pick}=$ $\left.x_{i j} \mid \xi_{j}, \theta_{i}\right)$ in Equation 1. All of these response probability functions are built from the logistic function,

$$
\operatorname{logistic}(l) \equiv \operatorname{logit}^{-1}(l) \equiv \frac{1}{1+\exp (-l)} .
$$


Details of the parameterizations are given here. A discussion of these item models more appealing to intuition is given in the next section.

\section{Dichotomous model}

The dichotomous response probability function can model items when there are exactly two possible outcomes. It is defined as,

$$
\begin{aligned}
& \operatorname{Pr}(\text { pick }=0 \mid a, b, g, u, \tau)=1-\operatorname{Pr}(\text { pick }=1 \mid a, b, g, u, \tau) \\
& \operatorname{Pr}(\text { pick }=1 \mid a, b, g, u, \tau)=\operatorname{logit}^{-1}(g)+\left(\operatorname{logit}^{-1}(u)-\operatorname{logit}^{-1}(g)\right) \frac{1}{1+\exp (-(a \tau+b))}
\end{aligned}
$$

where $a$ is the slope, $b$ is the intercept, $g$ is the pseudo-guessing lower asymptote expressed in logit units, $u$ is the upper asymptote expressed in logit units, and $\tau$ is the latent ability of the examinee (Birnbaum, 1968; Loken and Rulison, 2010). A \#PL naming shorthand has been developed to refer to versions of the dichotomous model with different numbers of free parameters. Model $n$ PL refers to the model obtained by freeing the first $n$ of parameters $b, a, g$, and $u$.

\section{Graded response model}

The graded response model is a response probability function for 2 or more outcomes (Samejima, 1969; Cai, 2010b). For outcomes $\mathrm{k}$ in 0 to K, slope vector $a$, intercept vector $b$, and latent ability vector $\tau$, it is defined as,

$$
\begin{aligned}
& \operatorname{Pr}(\text { pick }=K \mid a, b, \tau)=\frac{1}{1+\exp \left(-\left(a \tau+b_{K}\right)\right)} \\
& \operatorname{Pr}(\text { pick }=k \mid a, b, \tau)=\frac{1}{1+\exp \left(-\left(a \tau+b_{k}\right)\right)}-\frac{1}{1+\exp \left(-\left(a \tau+b_{k+1}\right)\right)} \\
& \operatorname{Pr}(\text { pick }=0 \mid a, b, \tau)=1-\operatorname{Pr}\left(\text { pick }=1 \mid a, b_{1}, \tau\right) .
\end{aligned}
$$

\section{Nominal model}

The nominal model is a response probability function for items with 3 or more outcomes (e.g., Thissen et al., 2010). It can be defined as,

$$
\begin{aligned}
a & =T_{a} \alpha \\
c & =T_{c} \gamma \\
\operatorname{Pr}\left(\text { pick }=k \mid s, a_{k}, c_{k}, \tau\right) & =C \frac{1}{1+\exp \left(-\left(s \tau a_{k}+c_{k}\right)\right)}
\end{aligned}
$$

where $a_{k}$ and $c_{k}$ are the result of multiplying two vectors of free parameters $\alpha$ and $\gamma$ by fixed matrices $T_{a}$ and $T_{c}$, respectively; $a_{0}$ and $c_{0}$ are fixed to 0 for identification; $s$ is the per-item slope; and $C$ is a normalizing constant to ensure that $\sum_{k} \operatorname{Pr}($ pick $=k)=1$.

\section{Item models}

Modern test theory employs item models, $\operatorname{Pr}\left(\right.$ pick $\left.=x_{i j} \mid \xi_{j}, \theta_{i}\right)$ (from Equation 1). To better appreciate how modern test theory works, it is helpful to develop an intuitive understanding of item models. The essential idea is the conversion of ordinal (or dichotomous) data into continuous data conditional on examinee skill. In Figure 1, the black dots represent the dichotomous data. Here we assume that examinee skill is known so that we can plot the black dots at the appropriate place on the $x$ axis. The next step is to partition the $x$ axis into equal interval bins. The propor-

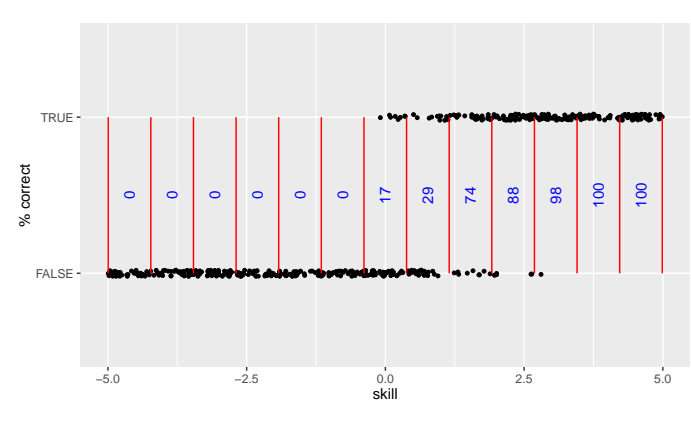

Figure 1: Dichotomous data converted into continuous data conditional on examinee skill. tion of examinees who responded correctly is displayed in blue in the middle of each bin. These blue numbers are our new continuous data, conditional on examinee skill. While we assumed that examinee skill was known, this assumption is actually unnecessary. The optimization algorithm can 
make a rough estimate of examinee skill, proceed to improve the model, and repeat this process until change is less than some epsilon.

To further inform your intuition about item models, it can be helpful to place yourself in the position of the optimization algorithm. Enter the following commands to launch the model explorer tool and browse to the output web server address. It is possible to do this without RStudio, but RStudio makes everything easier so we recommend using RStudio. Note that the port number (3726 printed below) may be different on your computer.

$>$ library (ifaTools)
$>$ itemModelExplorer()

Listening on http://127.0.0.1:3726

Your browser should show a screen similar to Figure 2. Try experimenting with all the controls. Early in the development of item models, model parameters closely corresponded to the psychological concepts of difficulty and discrimination (Birnbaum, 1968). For example, difficult items are only answered correctly by the brightest examinees while most examinees may correctly answer easy items. Discrimination quantifies how much we learn from a given response. Well-designed items discriminate examinee skill. The causes of poor item discrimination are many. An item may be hurt by awkward wording, by asking examinees something that is somewhat off-topic, or by asking the same question in slightly different ways.

Some item model parameters still retain a close connection to difficulty and discrimination. For example, the dichotomous model's $a$ parameter corresponds with discrimination and the negative $b$ parameter divided by $a$ corresponds with difficulty (Equation 5). However, as item models have grown more flexible, the parameter values and intuitive interpretation have become more distant. To understand item models in general, it is helpful to plot category curves and information by the latent trait (Figure 2). Some examples of latent traits which can be measured in this framework are mathematical skill, vocabulary, or sleep quality.

The way to interpret these plots is to start by picking a value for the latent trait. Suppose we know that examinee Alice has a latent ability of 2 logit units. If we trace across the plot where the $x$ axis is 2 then we find that Alice has a $75 \%$ chance of getting the item correct (blue curve) and a $25 \%$ chance of getting it incorrect (red curve). In addition, we find that this item will give us 0.05 units of information about Alice (black curve). The difficulty of the item is where the correct and incorrect curves cross at about 0.2 logits. The discrimination of the item is given by the information plot. This item provides more information about examinees with latent skill between -1 and 2 than elsewhere on the skill continuum.

Much can be gleaned about item models by inspection of these plots. However, it is worth conveying a few additional details specific to particular item models. The dichotomous model's $g$ and $u$ asymptote parameters are in logit units. To transform these values back into probabilities use R's plogis function. The $g$ parameter may represent the chance of an examinee guessing the item correctly. This parameter is also often called the pseudo-guessing parameter due to the probability of a low ability examinee getting an item correct at a non-zero asymptote. The $u$ parameter, or upper asymptote parameter, may represent the chance of an examinee committing a careless mistake, reflecting high ability examinee behavior. In this case, the upper asymptote never reaches one (Loken and Rulison, 2010).

By default, the nominal model uses trend for the T. a and T.c matrices (Equation 10). This parameterization is also known as the Fourier basis. The effect is that the alf and gam parameters control the lowest frequency variation to the highest frequency variation. To develop an intuition for how this works, set all parameters to zero then set a, alf1 and gam2 to 1 . Experiment with the gam parameters before you experiment with the alf parameters. Refer to Thissen et al. (2010) for discussion of the 


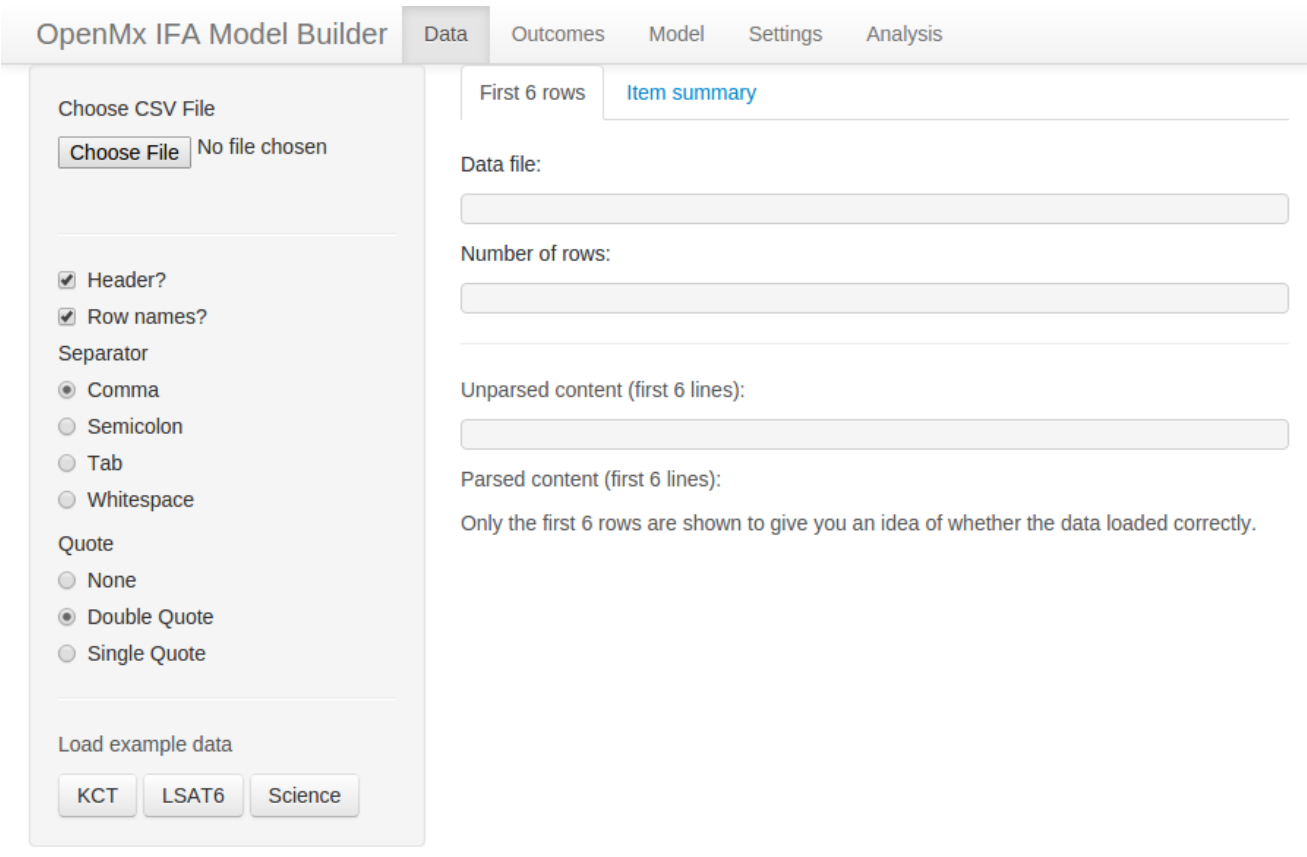

Figure 3: Initial screen shown after start up.

possibilities of this item model. Custom T. a and T.c matrices are not available in the model explorer app, but can be specified in $\mathrm{R}$ code.

The "Show/Edit parameters" checkbox has a special didactic purpose. Open two copies of the item model explorer. On one copy, un-check the "Show/Edit parameters" checkbox to hide the parameters and click the "Draw new parameters" button. On the second copy of the item model explorer, adjust the item model parameters to try to match the plot produced on the first item model explorer. You can check your answers by checking the "Show/Edit parameters" checkbox. When you play this game, you are doing part of what the optimization algorithm does when it fits models to data. Note that there is no need to practice this skill. The computer will do it for you.

\section{The model builder}

Enter the following commands to launch the model builder tool and browse to the output web server address. As before, it is possible to do this without RStudio, but RStudio makes everything easier so we recommend using RStudio. Note that the port number (3726 printed below) may be different on your computer.

\section{$>$ library (ifaTools) \\ $>$ modelBuilder()}

Listening on http://127.0.0.1:3726

Your browser should show a screen similar to Figure 3. Take care not to hit the Reload button because that will reset the app. Learn how to save your work (detailed below) before you experiment with the Reload button. Across the top are tabs that organize the major functions of the model builder app. On the left side is a control panel for the currently selected tab Data. Example data sets are available at the bottom of the control panel. You are welcome to experiment with these, but we will focus on the process of loading an external data set. If you prefer to follow along with a video then browse to http: //youtu. be/XHeb5_CWnCk for dichotomous data and http://youtu. be/iwtpleltteQ for polytomous data.

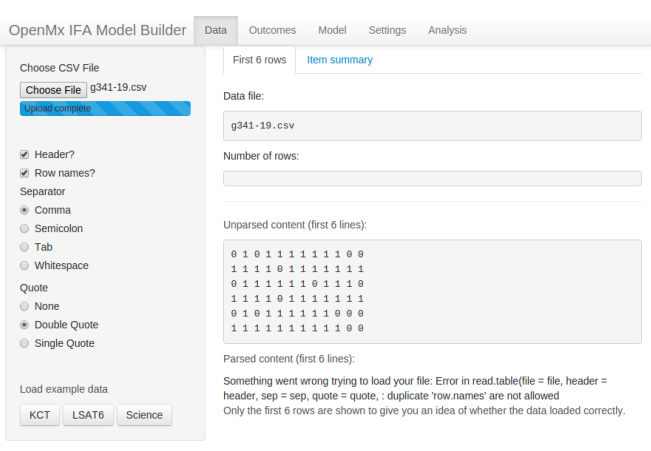

Figure 4: After loading the g341-19.csv data. 


\section{Dichotomous data}

Click on the "Choose File" button ${ }^{1}$ and select g341-19. csv, a dichotomous data set that is available in the ifaTools package (Pritikin, 2015a). The first 6 lines will appear in the "Unparsed content" section (see Figure 4). ${ }^{2}$ This makes it easy to see how the file is formatted. The "Parsed content" section reports an error. By reading the error carefully, you will find mention that "duplicate 'row.names' are not allowed." Since "Row names?" is enabled in the control panel, the model builder app expects the first column of data to be dedicated to row names. A row name is typically the examinee's name or numeric identifier. A glance at the unparsed content reveals that no row names have been given in this data set.

Click the "Row names?" checkbox in the control panel to disable row names. Immediately (without reloading the data), the error message in the "Parsed content" section will be replaced by some of the data organized into a single column. The column name will read X010111111100. A column name like that should raise suspicion. Since the "Header?" checkbox is enabled in the control panel, the model builder app expects the first line of the data to contain column names. Therefore, the first line of data is misinterpreted.

Click the "Header?" checkbox in the control panel to disable column names. The column in the "Parsed content" section will now be labeled V1. Click on the "Item summary" con-

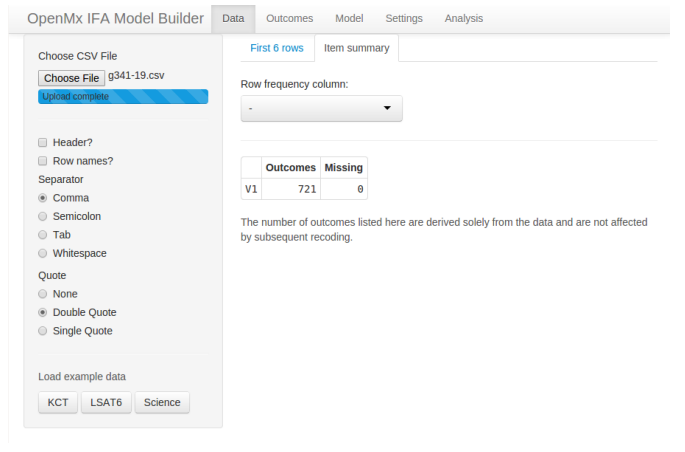

Figure 5: A summary of the g341-19. csv data set when parsed incorrectly as a single column. trol as an alternate way to verify that the data is loaded and parsed accurately. The main content area includes two elements, a selection for the "Row frequency column" and a table of items by Outcomes and Missing (see Figure 5). The "Row frequency column" selection is used when you have already reduced your data to unique rows and row counts. The example data set LSAT6 is in this format. For our current data set, leave "Row frequency column" set to -.

The information conveyed in the item table should rouse suspicion. There is only 1 row (or 1 item) with 721 outcomes. What happened is that the parsing is still not working and all the items are treated as a single column. For example, the first row "0 10111111100 " is not treated as 12 separate numbers but as a single uninterpreted unit. To fix the parsing, we need to select Whi tespace as the Separator in the control panel. With this last error corrected, the table is updated with 12 items labeled V1, V2, .., V12 and all with 2 outcomes. With the data correctly loaded, click on the "Outcomes" tab on the top bar.

The control panel on the "Outcomes" tab packs a lot of functionality (Figure 6). The first two selectors are mutually exclusive and permit working with all items having the same outcomes or with specific items, respectively. The outcome set "V1" is currently selected as seen in the control panel on the left side. In these data, all items have the same possible outcomes $(0$ or 1 ). Therefore, there is only one outcome set. The name "V1" does not just refer to the item "V1" but to all items, because all items have the same outcomes.

For clarity, it is often helpful to rename outcomes. The "Recode from" selector should have " 0 " selected. Change the to selector to <Rename>, enter "incorrect" for the "New name" value, and click the "Add mapping" button. This will create a recoding rule that will show up in the "Recode Table" output (Figure 7). Similarly, re-

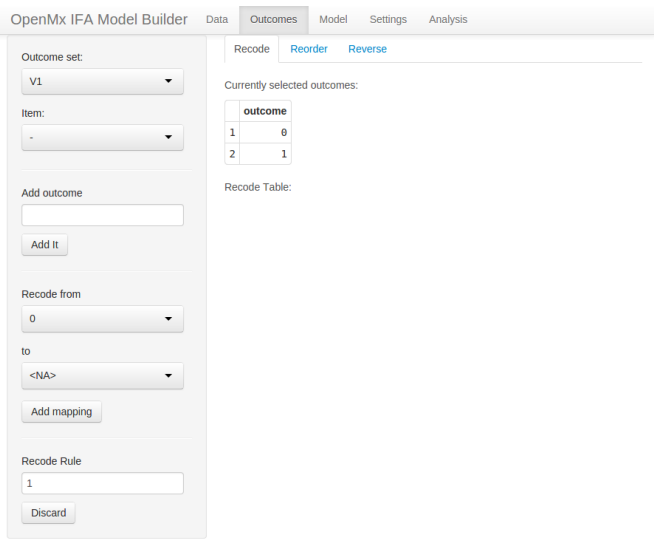

Figure 6: The Outcomes tab without any recoding rules. name the " 1 " outcome to "correct" and again

\footnotetext{
${ }^{1}$ It may read "Choose CSV File'." The exact appearance may differ on your system.

${ }^{2}$ We are aware that these screenshots are illegible when printed on paper. Inspect them using magnification on your computer.
} 
click the "Add mapping" button. At this point, you should have 2 rules in the "Recode Table" output.

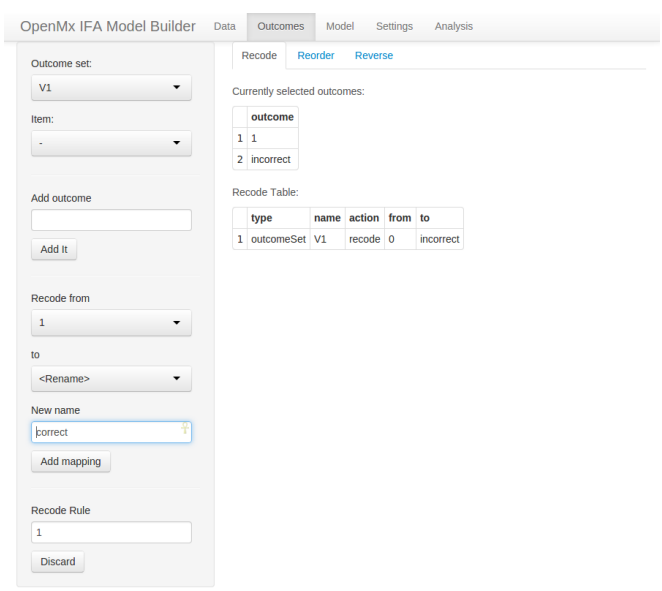

Figure 7: The outcome " 0 " is renamed to "incorrect" and we are poised to rename " 1 " to "correct." In a moment, we will click the "Add mapping" button.

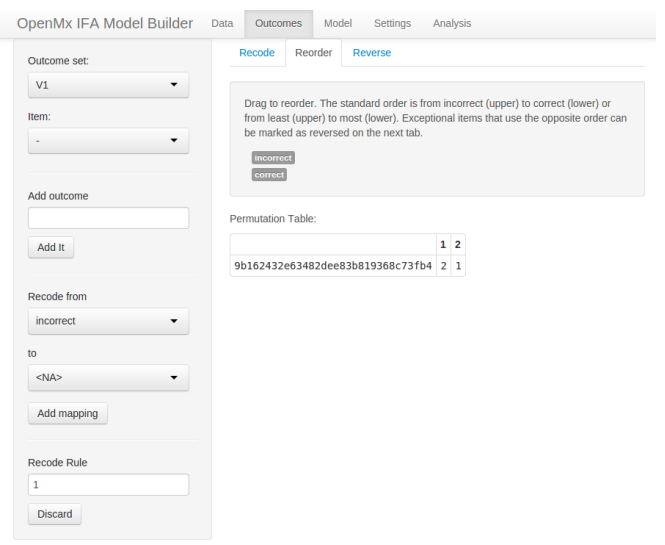

Figure 8: The outcome reorder tool with 1 reordering rule.

Click on the "Reorder" tab. Here you will find the outcomes sorted in lexical order. Drag one of the outcomes to reverse the order (as in Figure 8). Similar to the operation of renaming outcomes, this reordering step is not strictly necessary but is often helpful to keep things straight in our minds. With dichotomous outcomes, there are not that many ways that things can go wrong. However, it is good practice to use self-explanatory labels and standardized ordering. This is especially true when there are more than 2 outcomes to worry about.

We will not use the "Reverse" tab and other control panel elements in the present example. In survey design, it is common for outcomes to have a canonical order with some items reverse scored. The "Reverse" tab is used to reverse the outcome order of some subset of items without dragging the outcomes around with the "Reorder" tool. This can save a lot of dragging when there are more than 2 outcomes. The "Add outcome" tool permits the addition of outcomes that are not represented in the data. This might happen when a measure is in development and we are fitting a preliminary model just to get a vague idea of how the item is working. To fit an item that lacks data on some outcomes, it is usually necessary to use the nominal response model with a less than full rank parameterization (similar to Thissen et al., 1989). In addition to renaming, the recode mappings tool can merge or collapse outcomes. For example, we might have an outcome set consisting of "Agree," "Agree somewhat," "Not sure," "Disagree somewhat," and "Disagree." It is straightforward to merge "Agree somewhat" to "Agree" and "Disagree somewhat" to "Disagree," resulting in only 3 outcomes. Of course, it is not always obvious which outcomes to merge. The recode tool can also recode an outcome to $\langle\mathrm{NA}\rangle$, which causes that outcome to be interpreted as missing. Finally, the "Discard" button at the bottom of the control panel allows us to discard any rule that we created. So feel free to experiment.

Click on the "Model" tab on the top bar. The first decision we need to make is how many latent factors to include in our model (Figure 9). If we are not sure, a good starting point is 1 . By default, the first latent factor is called teacup. In case there was any doubt, "teacup" is not a very good name for a latent trait. We deliberately

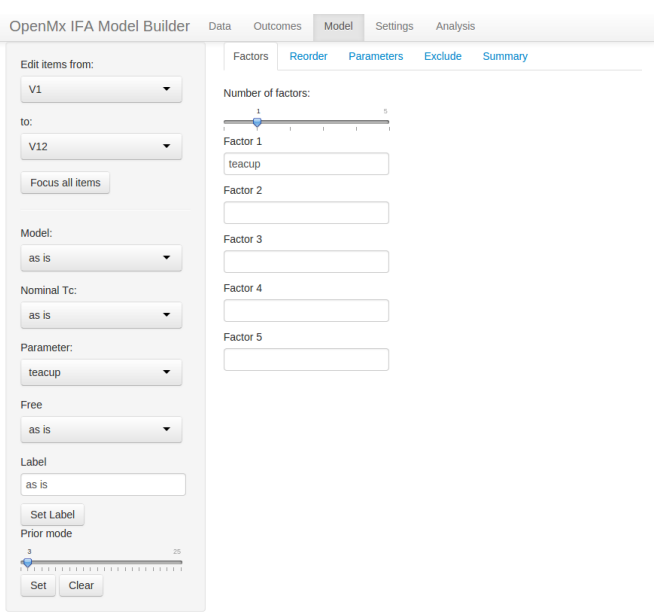

Figure 9: Configuration of latent factors. picked ridiculous factors names to encourage users to pick names that make sense in the context of the data under analysis. For example, a good factor name for a math test might be math. If you cannot think of a good factor name, you could use "latent trait," but this name only works well for a single factor model. You really should make an effort to think of descriptive names before you start using trait1, trait2, etc. If you are not sure how many 
factors to use then use 1 for now. We will revisit this question later.

The "Reorder" tab allows you to change the order of your items. This can be helpful because of the way that item model and parameter editing works. To get familiar with how item editing works, click on the "Parameters" tab. The main content area of the "Parameters" tab contains a lot of information. The first thing to notice is that all of the tables have the same column labels. Each item is assigned to a column. Using the control panel, we will focus on only a subset of items. Change the first selector "Edit items from:" from V1 to V7. This will hide the first 6 items, making the tables in the main content area look more manageable (Figure 10). The first two selectors facilitate item range selection. To reveal all items again, use the "Focus all i tems" button. Item selection is important to understand because the remainder of the controls in the control panel operate on only the selected (visible) items.

With only items V7 to V12 visible, just to demonstrate how it is done, let us place an equality constraint on the slope or latent factor loading. Type "slope" into the Label textbox and click the "Set Label" button. The label slope should appear in all columns of the first row of the Labels table in the main content area. Now let us switch to the first 6 items. This can be accomplished in a variety of ways. One way is to change the first selector from $V 7$ to $V 6$ and the second selector from $\mathrm{V} 12$ to $\mathrm{V} 1$.

With only items V1 to V6 visible, select drm from the "Model" selector. The value drm is an abbreviation for the 4PL dichotomous response model (Loken and Rulison, 2010), which has four parameters when there is one factor. The $g$ and $u$ rows should appear in all of the tables in the main content area. Parameter $g$ is the pseudoguessing lower bound and $u$ is the upper bound. The upper bound has been used to better model high ability examinees in a Computerized Adap-

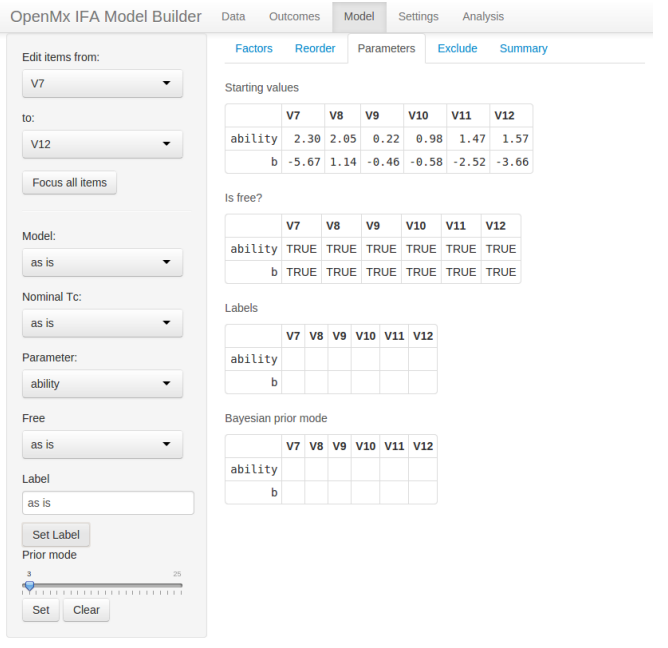

Figure 10: Editing the models and parameters of a tive Testing context (Magis, 2013). Even high ability examinees may occasionally miss an easy item. Here we will fix the upper bound to 1 (meaning that an examinee with sufficiently high ability will never answer incorrectly). Since the bound parameters are expressed on a logit scale, we will use logit(1). Select u from the "Parameter" selector and Inf from the "Free" selector (since logit(1) = inf). The row of "Starting values" for u should change to Inf and the corresponding "Is free" row should change from TRUE to FALSE. With this constraint, the 4PL dichotomous response model is equivalent to the 3PL model (Birnbaum, 1968).

The pseudo-guessing lower bound g represents the chance that an examinee will get the item correct by guessing. Typically, the expected guessed-correct probability for a 3-alternative item is $\frac{1}{3}$ and $\frac{1}{n}$ for an $n$-alternative item. Estimating the lower bound from data without telling the model a priori how many alternatives were presented typically requires much more data than is required to estimate other kinds of parameters. This is especially true for easy items because few participants will need to guess. It could be argued that easy items should have the lower bound set to a probability of zero. However, in an item set with some lower bounds fixed to zero and some free, the items with the lower bounds fixed to zero will provide more information than the items that take the chance of guessing into account. Therefore, we suggest fixing the lower bound to $\frac{1}{n}$ for an $n$-alternative

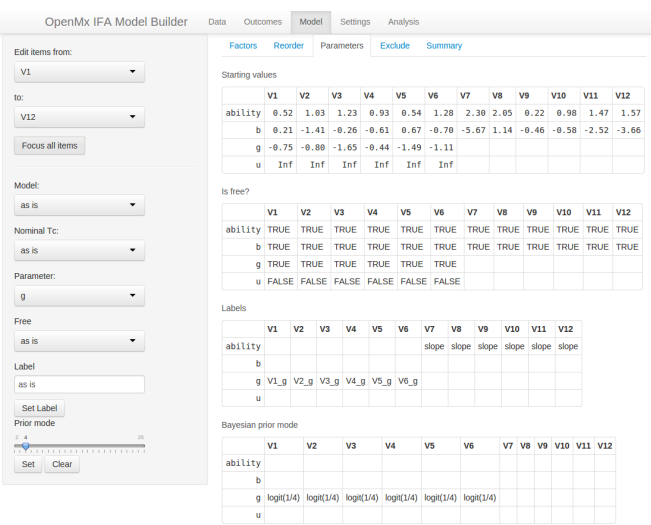

Figure 11: Item tables after setting up our model. item when estimation of the lower bound is not of interest.

As a compromise between fixing and freeing, a Bayesian prior can be used with the mode of the prior set to the expected probability. While some researchers are uneasy about the use of priors (Gelman, 2008), the practice is not new (e.g, Baker and Kim, 2004, Chapter 7). The prior ensures that a parameter will converge even when there is not enough data to estimate it, but at the same time, 
the model retains some flexibility to adapt to unexpected data. To set a prior, drag the "Prior mode" slider and click the "Set" button. Let us imagine that these items had 4 alternatives. Select $g$ from the Parameter selector, move the "Prior mode" slider to 4, and click the nearby Set button. Two tables will change. Each cell of the g row of the Labels table will be assigned a unique label. This is necessary because Bayesian priors are associated with labels, not with particular parameters. In addition, the "Bayesian prior mode" table will show logit $(1 / 4)$ in the $g$ row. The logit usage reflects that the parameter is estimated on the logit scale, but it is much easier for humans to understand a probability expressed as a fraction rather than raw logit units.

We will not use the "Nominal Tc" selector for these data. "Nominal Tc" only applies to items with more than 2 possible outcomes when using the nominal response model (Thissen et al., 2010). Before proceeding, go ahead and click the "Focus all items" button. Your screen should look like Figure 11, except for different starting values. Click on the "Exclude" tab. This is an easy way to exclude some of the items from analysis. Finally, click on the "Summary" tab. Here you will find a summary of your model settings. Note that the number of outcomes may differ from the number of outcomes reported in the summary table found on under the "Data" top bar page due to recoding.

We are done setting up our model. Before proceeding, it would be wise to save our model configuration so we can come back at a later time and make small adjustments without going through the whole exhaustive process again. Click Settings on the top bar. In the main content area, you will find a preview of what the settings file will look like. Click the "Download" button and move the file to a suitable location on your computer.

To verify that you can reliably restore the saved settings, open a new browser tab to the same address by pasting the URL address from the current tab (without closing the current one). You should get a screen like Figure 3. Again go through the procedure of loading the data

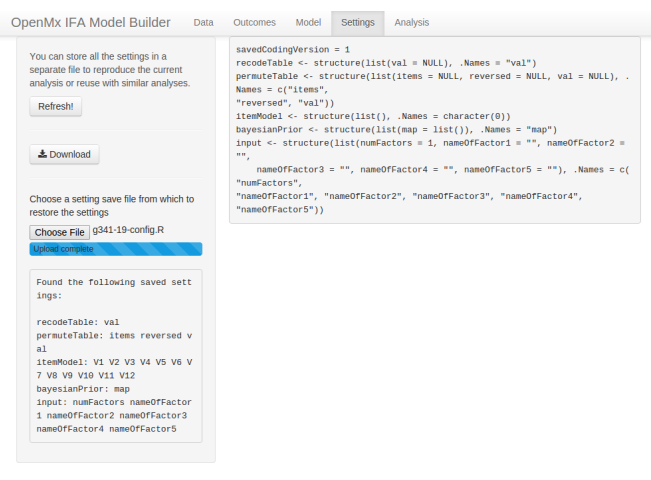

Figure 12: Restoring the settings. (Figures 4 and 5). Once your data is loaded, click Settings on the top bar and load the file that you recently saved. If all goes well, you should see a screen similar to Figure 12. Go ahead and look back through the editors under the Outcomes and Model sections of the top bar. You should find all your hard work faithfully preserved. Now you can close either of the 2 browser tabs that you have open. They both have the same status.

With our model set up and saved, click Analysis on the top bar. This screen looks and functions similarly to the Settings screen. However, the control panel offers a few options specific to code generation. The "Functional form for dichotomous bound prior density" selector chooses the distributional form of the Bayesian prior. Logit-normal is the more recent scheme (Cai et al., 2011). The "Information matrix method" control is set to Oakes by default. In a simulation study included with OpenMx, the Oakes method (Oakes, 1999) exhibited accuracy comparable to central difference with Richardson extrapolation and time linear in the number of parameters. Click the "Download" button and save the Rmarkdown code. The Rmarkdown file and your data need to be in the same directory. Either move the Rmarkdown file to your data directory, or alternately, you can specify a full path in the read.csv statement (lines 16-17). Open the file in RStudio and click the "Knit HTML" button.

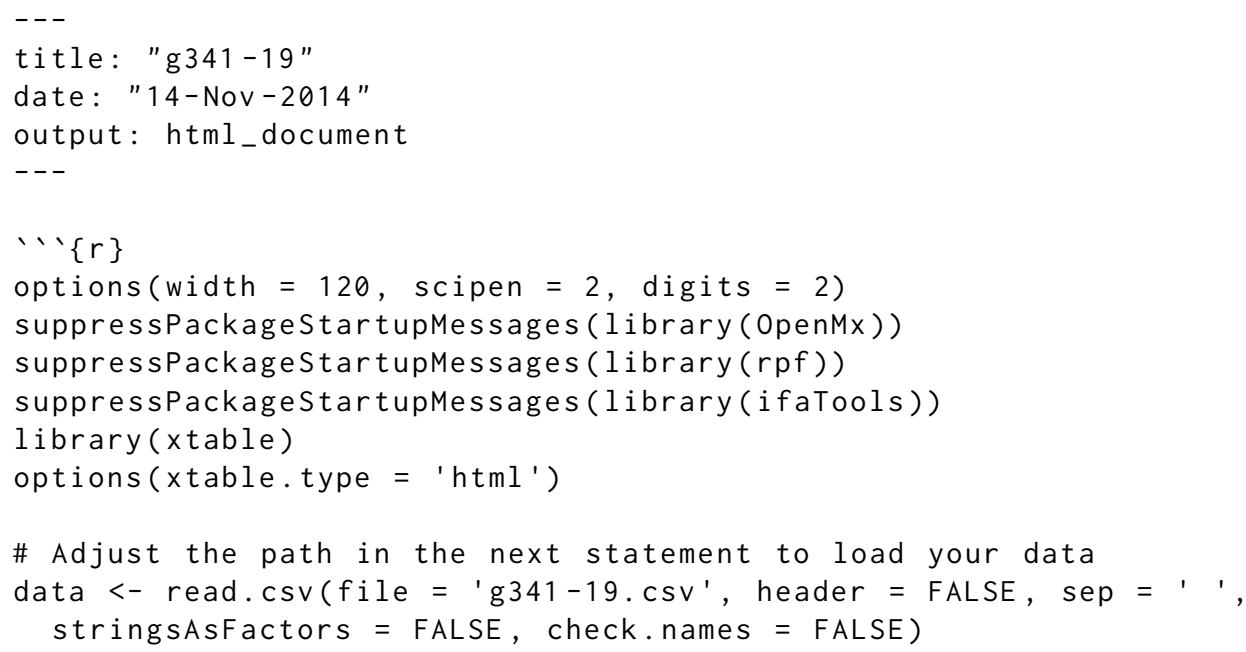




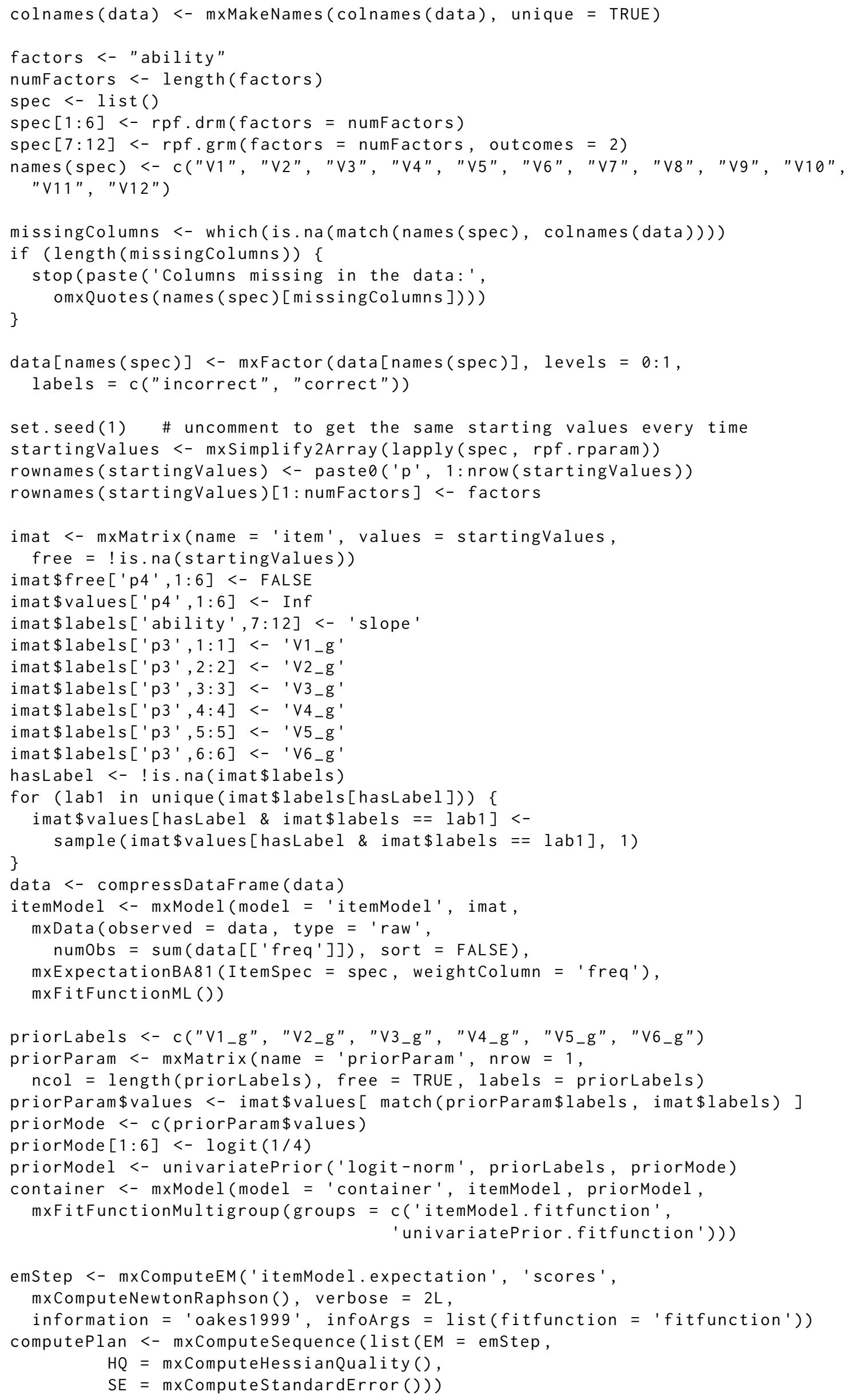




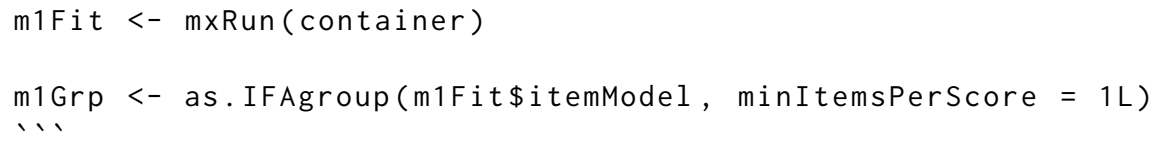

The details of the generated report are likely to evolve. There may be some differences between the generated code in this article and the most recent version, but there should be a correspondence between the basic elements. The first chunk of code builds the model and optimizes it. We adjust the output of long lines and numbers (line 8) and load packages (lines 9-13). The data set is loaded (Figure 5) in lines 16-17. Latent factors are configured (Figure 9) in lines 20-24. We strongly encourage the use of informative column (item) names, but line 18 will take care of assigning syntactically valid column names if informative names are not available. The script is crafted such that it can work on other data sets as long as the required columns are found (line 28). mxFactor does the recoding and reordering (Figures 6-8). mxFactor offers a number of important safety and convenience features beyond what is available from factor or ordered (line 34). The item mxMatrix (line 43) contains most of the information in the item tables (Figure 11). Everything goes into the container model (line 72). The model is optimized (line 83). Since we did not disable "Show model fitting progress" (reflected by verbose $=2 \mathrm{~L}$ at line 77), we obtain some diagnostics upon knitting the Rmarkdown to HTML.

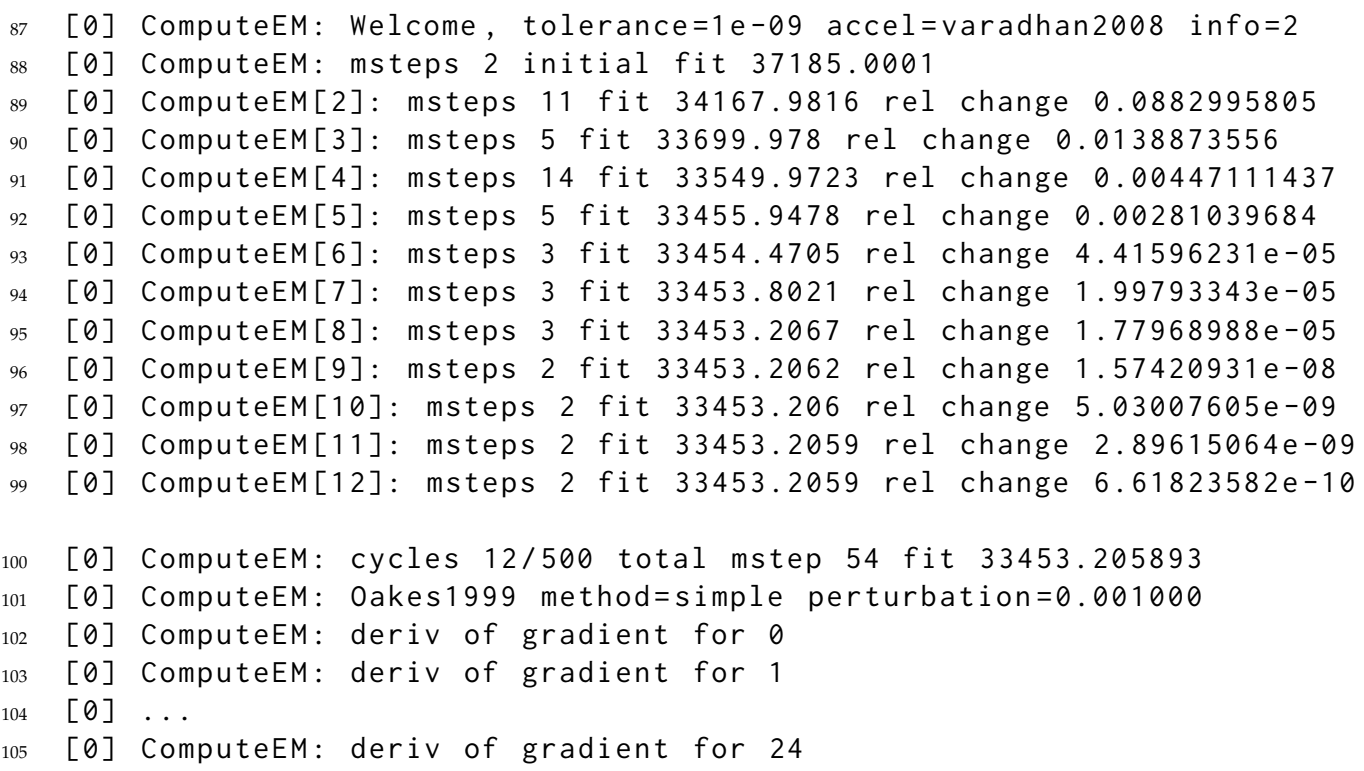

Given that the starting values are random, the initial fit and trajectory (lines 88-99) should differ when you try optimizing the same model but the optimum (line 100) should be the same to within $10^{-2}$. If you do not reach the same solution, try again with different starting values. At the time of writing, optimization is faster on multicore CPUs running on operating systems other than Microsoft Windows. As soon as Windows supports OpenMP then we expect performance differences between operating systems to narrow.

The function as. IFAgroup (line 85) is a bridge between OpenMx and rpf (Pritikin, 2015b). The rpf name is an acronym for response probability function and contains many IFA-specific diagnostic functions. In addition, rpf can be used to analyze IFA models optimized by packages other than OpenMx. This modularity facilitates the comparison of parameter estimates between packages. While most of the code discussed so far is related to OpenMx, the remainder of this report will mostly involve rpf.

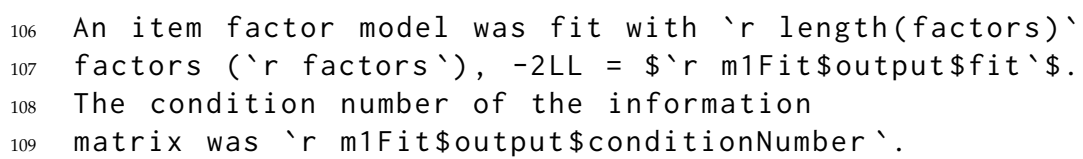

This is a boilerplate report of model fit. It renders as, "An item factor model was fit with 1 factors (ability), $-2 \mathrm{LL}=33453.21$. The condition number of the information matrix was 70.91." It is not really feasible to generate a complete Results section because there are always considerations idiosyncratic to a particular project that dictate how the Results section should best unfold. However, it is likely that some additional boilerplate reporting will be added to the model builder app in a future release.

Although IFA models consider an examinee's response pattern as the unit of analysis, the sumscore is often of chief practical importance. For example, students taking the Standardized Aptitude 


\begin{tabular}{rrrrrrrrrrrr}
\hline & V1 & V2 & V3 & V4 & V5 & V6 & V7 & V8 & V9 & V10 & V11 \\
\hline V2 & 0.1 & & & & & & & & & & \\
V3 & -0.0 & -2.6 & & & & & & & & & \\
V4 & 1.7 & -0.1 & 1.2 & & & & & & & & \\
V5 & -0.6 & -0.9 & -1.3 & 5.1 & & & & & & & \\
V6 & 0.2 & 0.1 & -0.3 & 0.3 & -1.1 & & & & & & \\
V7 & 0.1 & 6.4 & 0.6 & 2.4 & 0.3 & 5.0 & & & & & \\
V8 & -0.5 & -0.3 & -0.7 & -4.0 & -0.2 & 0.1 & 3.9 & & & & \\
V9 & 3.6 & 10.7 & 1.1 & 2.6 & 1.8 & 5.8 & 37.1 & 0.3 & & & \\
V10 & -2.0 & 9.1 & 0.3 & -0.3 & 0.1 & -0.2 & 10.2 & -0.5 & 16.2 & & \\
V11 & -1.0 & -11.5 & -2.6 & -1.1 & -0.6 & -1.4 & -1.9 & -4.8 & -0.5 & -0.7 & \\
V12 & -0.1 & -1.7 & 3.9 & -2.9 & -1.9 & -0.7 & 0.8 & -2.0 & -0.1 & 0.6 & -7.1 \\
\hline
\end{tabular}

Table 1: $\log p$-value of local dependence between item pairs.

Test for college admission only receive their sum-score and do not even know which items they answered correctly or incorrectly (unless they earned the maximum sum-score). The observation that the sum-score is important has lead to the development of a family of diagnostic tests that examine how well an IFA model predicts sum-scores.

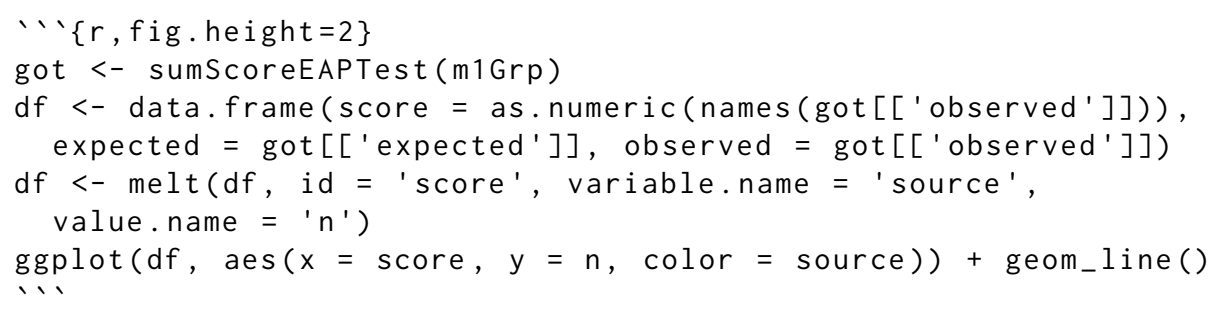

The first plot provides an overview of how all the items work together to predict sum-scores (Figure 13). sumScoreEAPTest also conducts a statistical test to produce a $p$-value, but this is not reported here because the test is fairly new and the meaning of the test has not yet been well established (Li and Cai, 2012). However, it is still worth looking at this plot because you might notice something that is obviously wrong with the

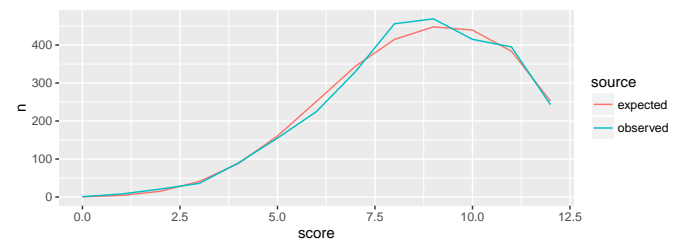

Figure 13: Comparison of the predicted and observed sum-scores. model (i.e., if the curves mismatch drastically).

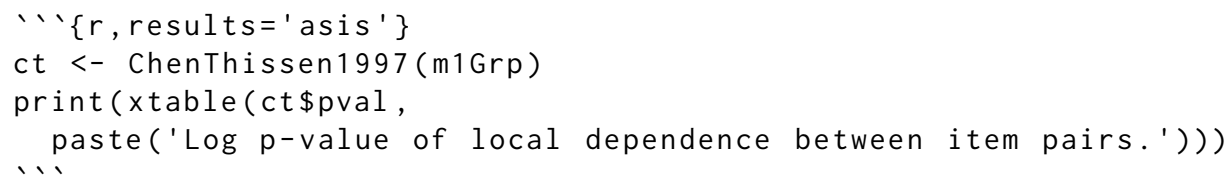

A test of local dependence is important to examine, as it is an assumption of IFA (Yen, 1993). Table 1 exhibits the $\log p$-value of the null hypothesis that there is no local dependence between item pairs. Since $\log (.01) \approx-4.6$, any absolute magnitude greater than 4.6 can be interpreted as rejecting the null hypothesis at the .01 level. The sign of each $p$-value is determined by ordinal gamma, a measure of association (Agresti, 1990). Positive numbers indicate more correlation than expected. These are cause for concern and suggest local dependence (Chen and Thissen, 1997). Negative numbers indicate less correlation than expected. Table 1 is also a good example of a weakness of comparing expected and observed frequencies: all you can know is that something is suboptimal, but not specifically what. The local dependence is most severe between item pairs V7/V9, V9/V10, and V2/V9. Item pair V2/V11 also has a large magnitude value, but this is less of a concern because the sign is negative. Unfortunately, this diagnostic only reveals potential deficiencies, but does not suggest how to address them. Improvement of the model (or the items) often requires some guesswork and trial-and-error.

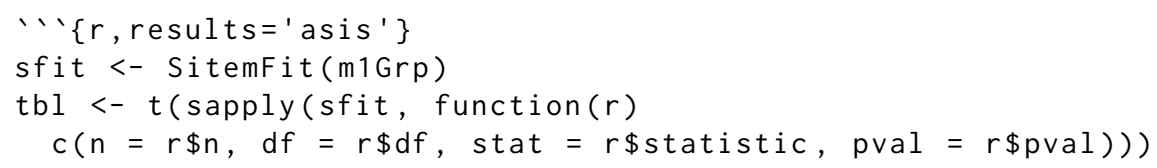




\begin{tabular}{rrrrr}
\hline & $\mathrm{n}$ & $\mathrm{df}$ & statistic & $\log p$-value \\
\hline V1 & 2844 & 8 & 6.58 & -0.54 \\
V2 & 2844 & 8 & 7.30 & -0.68 \\
V3 & 2844 & 8 & 7.17 & -0.66 \\
V4 & 2844 & 8 & 10.12 & -1.36 \\
V5 & 2844 & 8 & 19.00 & -4.21 \\
V6 & 2844 & 8 & 8.50 & -0.95 \\
V7 & 2844 & 9 & 33.45 & -9.10 \\
V8 & 2844 & 9 & 5.48 & -0.24 \\
V9 & 2844 & 9 & 34.42 & -9.49 \\
V10 & 2844 & 10 & 12.61 & -1.40 \\
V11 & 2844 & 8 & 43.06 & -13.97 \\
V12 & 2844 & 8 & 20.20 & -4.64 \\
\hline
\end{tabular}

Table 2: Sum-score item-wise fit.

${ }_{127}$ print(xtable(tbl, paste0('Sum-score item-wise fit.' ))

Sum-score item fit is another tool for model assessment (Orlando and Thissen, 2000; Kang and Chen, 2008). Each item is tested against the null hypothesis that the other items accurately predict the outcome of the item of interest (Table 2). Again $p$-values are in log units so a magnitude larger than 4.6 is significant at the .01 level. In contrast to the test for local dependence, the sign of the $p$-value does not mean anything special here. The column $n$ is included for data sets with missingness. When there is missingness, it can be advantageous to exclude the item with the most missing values to increase the sample size of the test. Refer to the Si temFit help for details on the options for missing data.

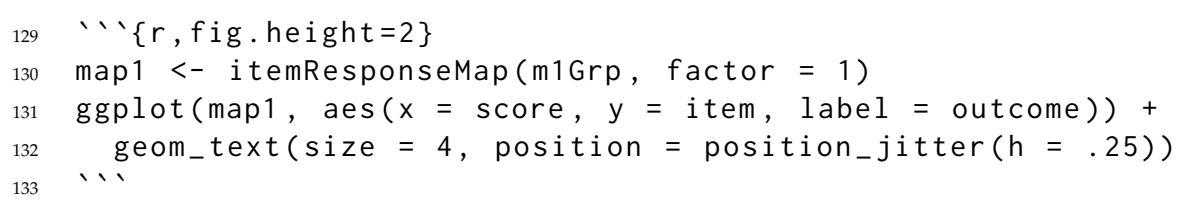

An item response map is a crude tool for diagnosing certain model misspecifications (Figure 14). Each outcome is assigned the average latent score of all the examinees who picked that outcome. Usually we advocate the use of the actual outcome labels (e.g., "incorrect" and "correct") instead of numbers. For this plot, however, we make an exception because the numbers make it easy to inspect whether the outcomes are in ascending order. If descending order is observed then it is worth checking whether the

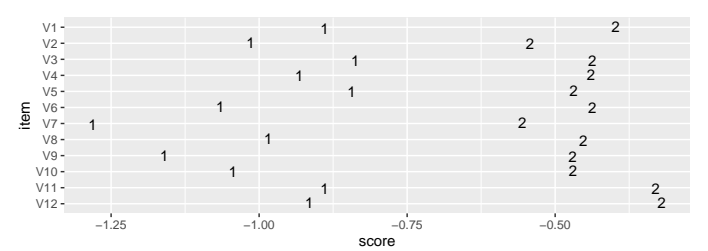

Figure 14: Item outcome by average latent score. item needs to be reverse scored or to consider whether the item was misinterpreted by some examinees. If the response data were manually collected then the data entry process should also be checked for errors.

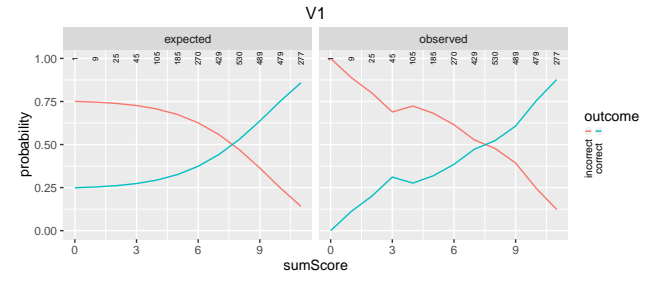

Figure 15: Expected and observed outcome by sum-score.

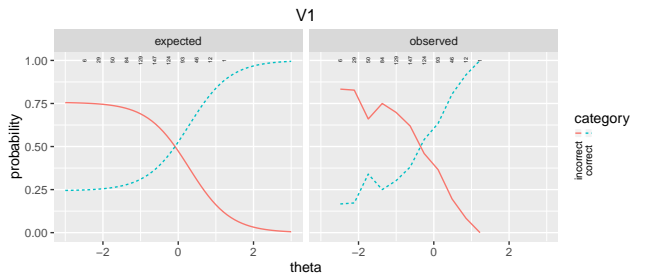

Figure 16: Expected and observed outcome by latent score.

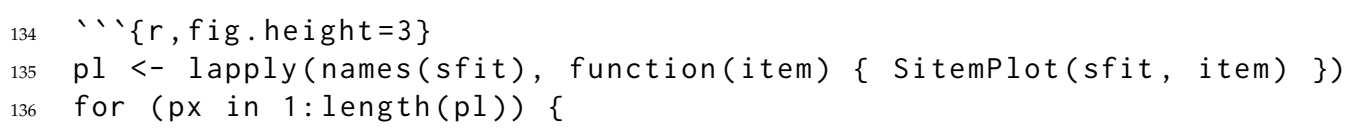




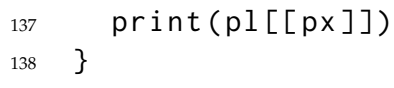

Two approaches are available to plot response probability functions against a latent trait. The same ingredients that go into the production of Table 2 can also be plotted (Figure 15). A similar plot can be obtained by plotting the outcomes probabilities against the latent trait. This is known as an item characteristic curve plot (Figure 16). The main advantage of Si temPlot over iccPlot is that Si temPlot is one-dimensional regardless of the number of latent factors. With iccPlot, you must pick a basis vector in the latent space. The tiny numbers across the proba-

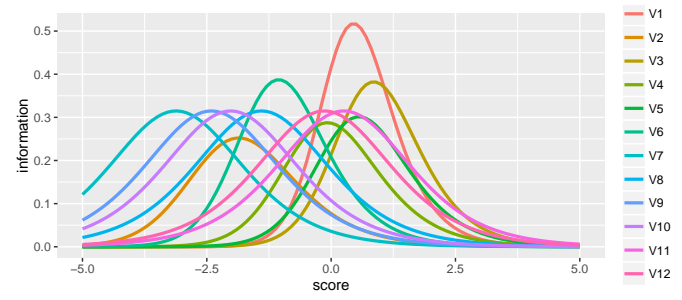

Figure 17: Item information by latent score. bility $=1$ line of Figures 15 and 16 are the sample size at that point on the $x$ axis.

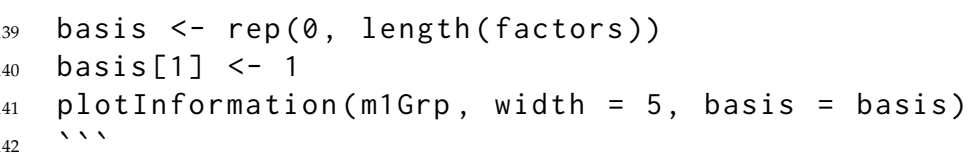

Figure 17 exhibits item information by latent score. Similar to iccPlot, this plot requires the selection of a basis vector when there is more than 1 latent factor. Notice that items V7-V12 peak at the same height (near 0.31). This is due to our equality constraint on the slope or factor loading on these items. By placing this constraint, we assume a priori that each of these items contributes exactly the same amount of information.

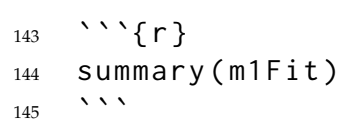

Summary of container

free parameters:

\begin{tabular}{|c|c|c|c|c|c|}
\hline name & matrix & row & col & Estimate & Std.Error \\
\hline itemModel.item $[1,1]$ & itemModel.item & ability & V1 & 1.82 & 0.278 \\
\hline itemModel.item $[2,1]$ & itemModel.item & p2 & V1 & -0.51 & 0.230 \\
\hline V1_g & itemModel.item & p3 & V1 & -1.14 & 0.208 \\
\hline itemModel.item[1,2] & itemModel.item & ability & V2 & 1.24 & 0.119 \\
\hline itemModel.item $[2,2]$ & itemModel.item & $\mathrm{p} 2$ & V2 & 2.58 & 0.140 \\
\hline V2_g & itemModel.item & p3 & V2 & -1.27 & 0.337 \\
\hline itemModel.item $[1,3]$ & itemModel.item & ability & V3 & 1.56 & 0.261 \\
\hline itemModel.item $[2,3]$ & itemModel.item & p2 & V3 & -1.03 & 0.272 \\
\hline V3_g & itemModel.item & p3 & V3 & -1.16 & 0.192 \\
\hline itemModel.item $[1,4]$ & itemModel.item & ability & V4 & 1.36 & 0.161 \\
\hline itemModel.item $[2,4]$ & itemModel.item & $\mathrm{p} 2$ & V4 & 0.41 & 0.158 \\
\hline V4_g & itemModel.item & p3 & V4 & -1.10 & 0.277 \\
\hline itemModel.item $[1,5]$ & itemModel.item & ability & V5 & 1.41 & 0.196 \\
\hline itemModel.item $[2,5]$ & itemModel.item & p2 & V5 & -0.47 & 0.203 \\
\hline V5_g & itemModel.item & p3 & V5 & -1.03 & 0.203 \\
\hline itemModel.item $[1,6]$ & itemModel.item & ability & V6 & 1.50 & 0.130 \\
\hline itemModel.item $[2,6]$ & itemModel.item & p2 & V6 & 1.84 & 0.119 \\
\hline V6_g & itemModel.item & p3 & V6 & -1.43 & 0.317 \\
\hline slope & itemModel.item & ability & V7 & 1.12 & 0.037 \\
\hline itemModel.item $[2,7]$ & itemModel.item & p2 & V7 & 3.50 & 0.097 \\
\hline itemModel.item $[2,8]$ & itemModel.item & p2 & V8 & 1.57 & 0.056 \\
\hline itemModel.item $[2,9]$ & itemModel.item & p2 & V9 & 2.70 & 0.075 \\
\hline itemModel.item $[2,10]$ & itemModel.item & $\mathrm{p} 2$ & V10 & 2.27 & 0.066 \\
\hline itemModel.item $[2,11]$ & itemModel.item & $\mathrm{p} 2$ & V11 & -0.28 & 0.047 \\
\hline itemModel.item $[2,12]$ & itemModel.item & p2 & V12 & 0.15 & 0.04 \\
\hline
\end{tabular}

observed statistics: 720

estimated parameters: 25

degrees of freedom: 695 
fit value ( $-2 \ln$ units ): 33453

number of observations: 2844

Information Criteria:

\begin{tabular}{rrr|r} 
& I df Penalty | Parameters Penalty | Sample-Size Adjusted \\
AIC: & 32063 & 33503 & NA
\end{tabular}

BIC: $\quad 27926 \quad 33652 \quad 33573$

To get additional fit indices, see help(mxRefModels)

timestamp: 2016-02-24 10:14:59

Wall clock time (HH:MM:SS.hh): $00: 00: 02.72$

OpenMx version number: 2.3.1.254

Need help? See help(mxSummary)

Exhibited above is the OpenMx provided summary of model fit. IFA models are exponential family models so we obtain AIC and BIC. More fit statistics are available if we provide the saturated and independence reference models. Reference models will be requested in our next example.

\section{Polytomous data}

Since many things are common between dichotomous and polytomous items, we will move quickly through the process of model set up and result interpretation. Click on the "Choose File" button and select preschool.csv, a data set from Thissen and Steinberg (1988) available in the ifaTools package. Click the "Row names?" checkbox in the control panel to disable row names. The format of these data are closer to what is expected by default than our first example so less fiddling is required. Click on the "Item summary" tab. Here it appears that there are 3 items, but the freq column is not an item. freq indicates how many times a row appeared in the original data. These data are compressed; only unique rows are provided with frequency counts. To

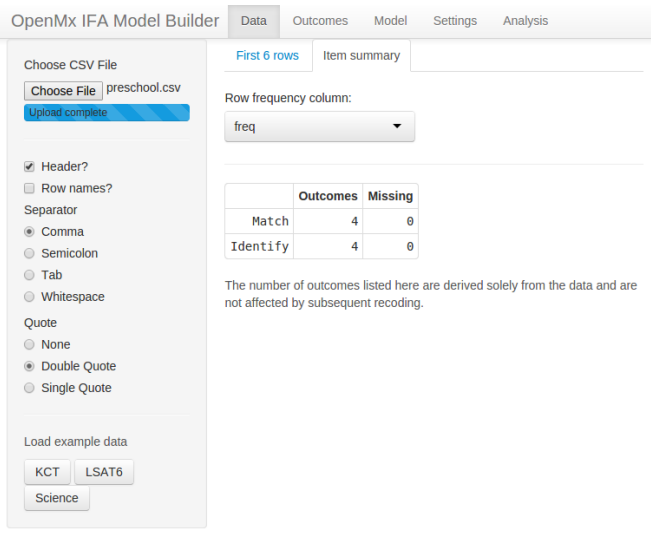

Figure 18: Data with a row frequency column. instruct the model builder to interpret the freq column as frequency counts, select freq from the "Row frequency column" selector (Figure 18).

This data set is from a preschool test of numerical knowledge. Each item is actually a combination of 2 dichotomous items. Similar questions were asked regarding the number 3 and the number 4 and the pattern of responses mapped to an outcome code. The outcomes should be renamed with the recoding tool under the "Outcomes" tab on the top bar (recall Figure 7). Outcomes 0, 1, 2, and 3 should be renamed to "neither," "3 only," "4 only," and "both correct," respectively, using the "Recode" tab under the Outcomes top bar page. After renaming, reorder the items into the correct order (Figure 19).

Click Model on the top bar. On the "Factors" tab, we will name the single latent factor "math." Switch to the Parameters tab. Here we select nrm from the "Model" selector. The acronym "nrm" stands for the nominal response model (Thissen et al., 2010). This parameterization of the nominal model can accommodate basis matrices $T_{a}$ and $T_{c}$ to customize the meaning of the slope and intercept coefficients, respectively. In principle, the basis matrices can take any pattern, but the model builder app is limited to a Fourier basis (a.k.a. trend basis) for the $T_{a}$ matrix and a small number of options for the $T_{\mathcal{C}}$ matrix.

With $T_{a}$ set to the trend basis, we cannot free both math and alf1 because they have the same effect on the model and would cause the model to be unidentified. Fix alf1 to 1 . Select alf 1 from the "Parameter" selector and select 1 from the "Free" selector. Since we have worked with this data set already, we know a few things that can give us a more parsimonious model. The alf2 parameters can be set equal since both items exhibit poor discrimination between neither, 3 only, and 4 only but good discrimination between these outcomes and both correct. Select alf2 with the "Parameter" selector and set the label to eq1. Since both items are equally difficult, we can equate gam1. Select gam1 with the "Parameter" selector and set the label to eq2. To avoid overfitting with the highest frequency basis vector, fix gam 3 to 0 . Select gam 3 with the Parameter selector and select $\theta$ with the Free selector. Figure 20 exhibits the final parameter settings.

Click Analysis on the top bar. Ensure that "Fit reference models" is selected, and download the analysis script. The Rmarkdown file and your data need to be in the same directory. Either move the Rmarkdown file to your data directory, or alternately, you can specify a full path in the read.csv 


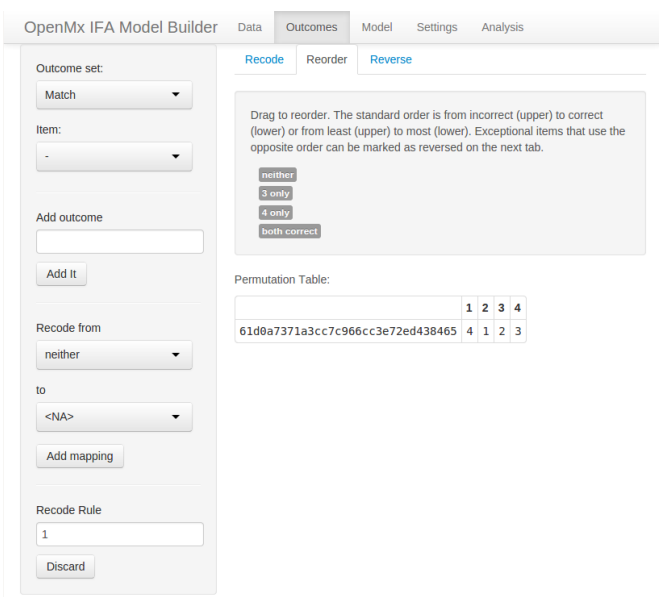

Figure 19: Outcomes renamed and reordered.

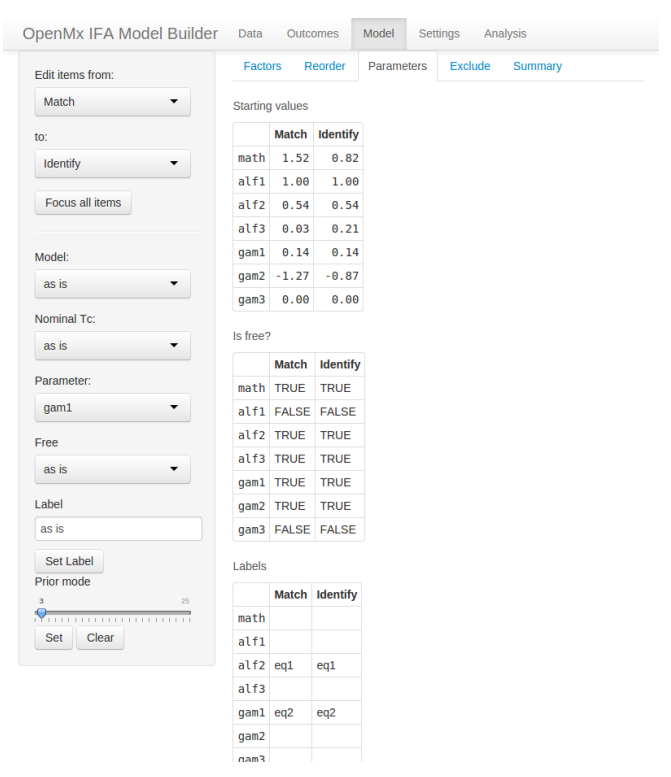

Figure 20: Item model and parameter configuration with equality constraints.

statement (line 162). Open the file in RStudio and click the "Knit HTML" button. Although this is a simple model, it can take almost 100 E-M cycles to converge. Therefore, we omit reproduction of the diagnostic output issued during model fit.

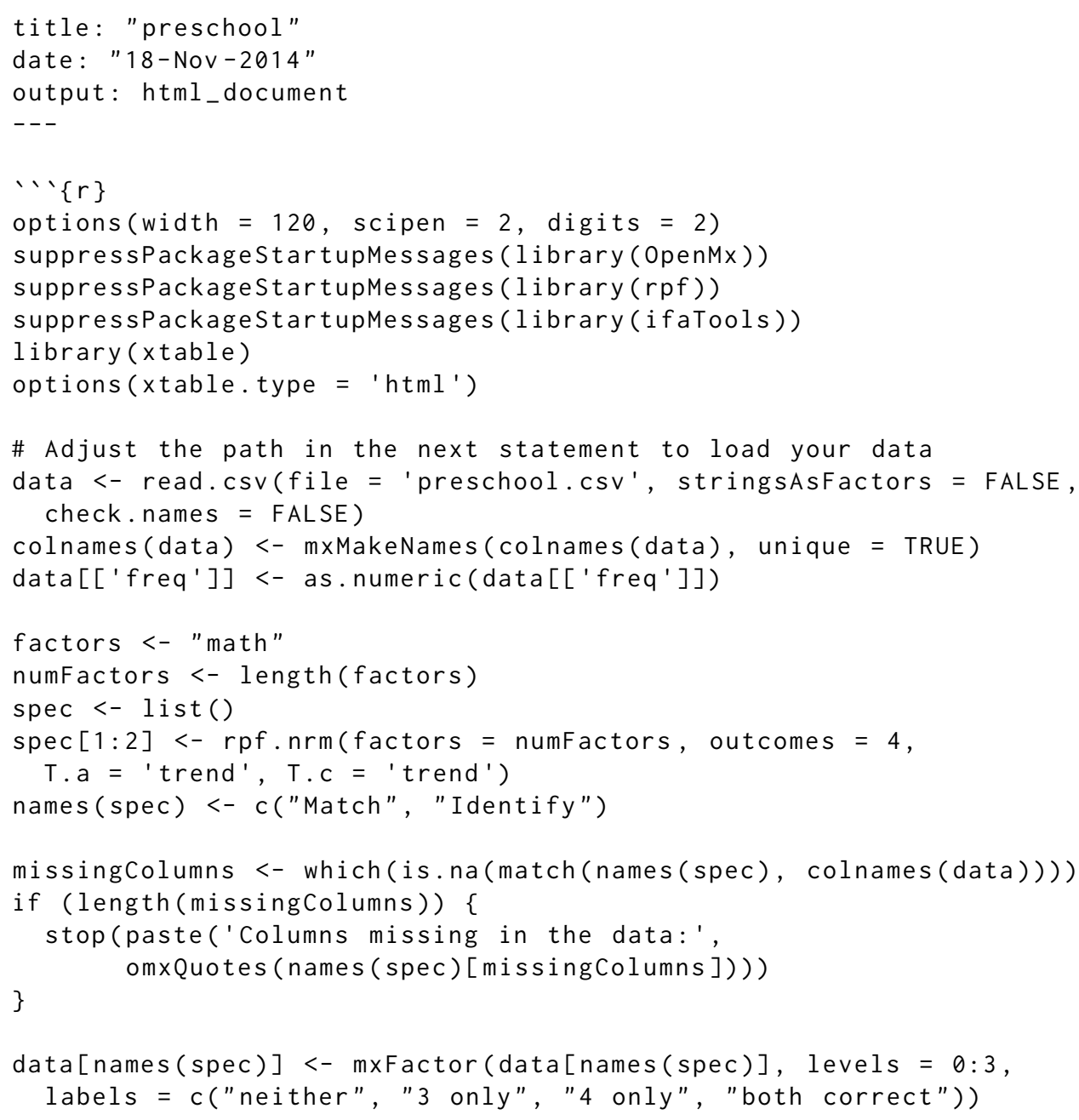




\begin{tabular}{rrrrr}
\hline & $\mathrm{n}$ & $\mathrm{df}$ & statistic & $\log p$-value \\
\hline Match & 592 & 7 & 9.46 & -1.51 \\
Identify & 592 & 7 & 9.33 & -1.47 \\
\hline
\end{tabular}

Table 3: Sum-score item-wise fit.

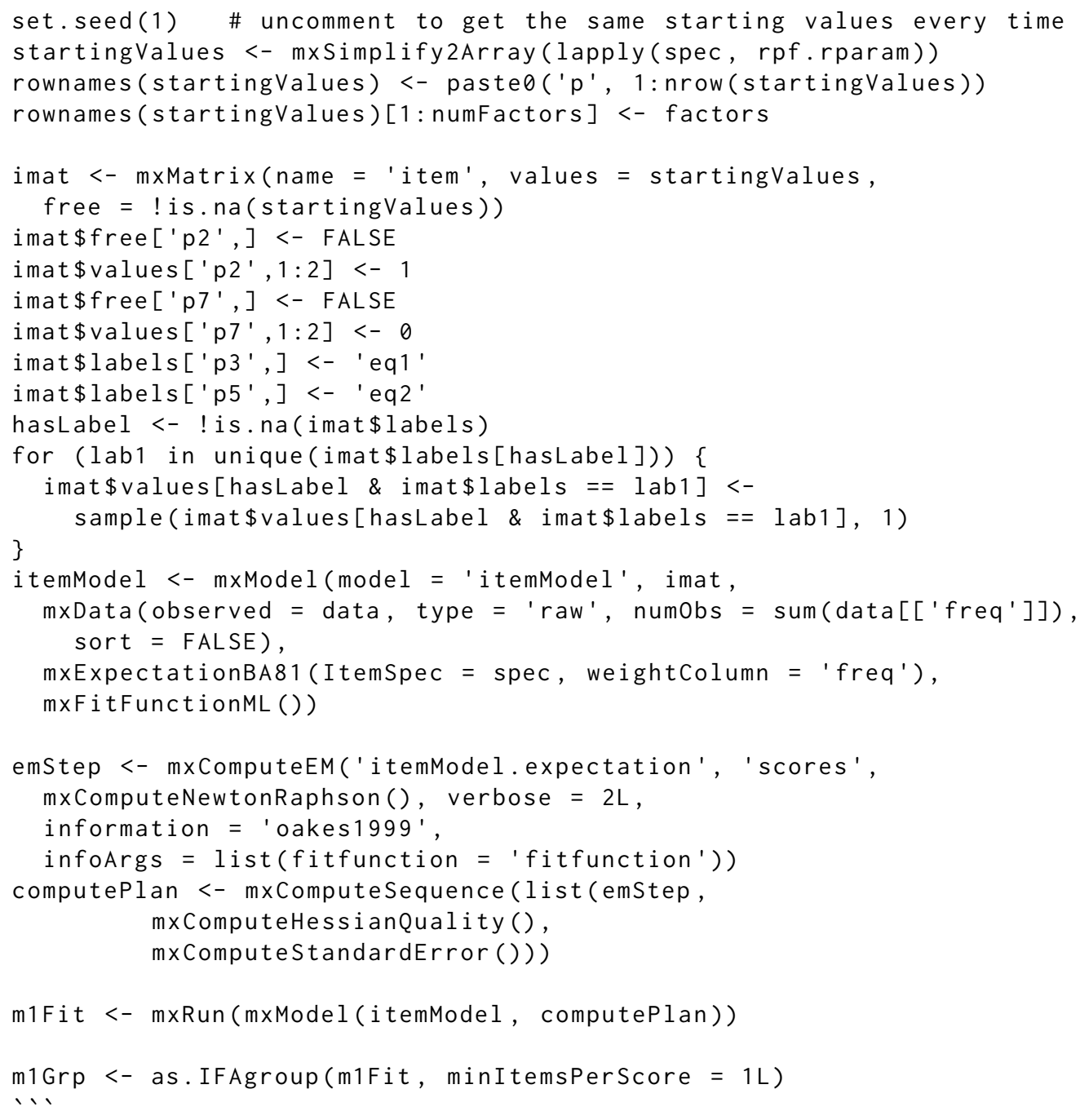

Although response pattern frequencies are typically natural numbers, fractional frequencies are not prohibited (line 164). A Fourier basis is used for both nominal model transformation matrices (line 170). Customization is limited in the model builder app, but you can use any matrices by editing the generated code. Starting values must respect equality constraints (line 197). By default OpenMx, sorts data prior to optimization. Since our data are already compressed, no benefit would be obtained by sorting (line 202).

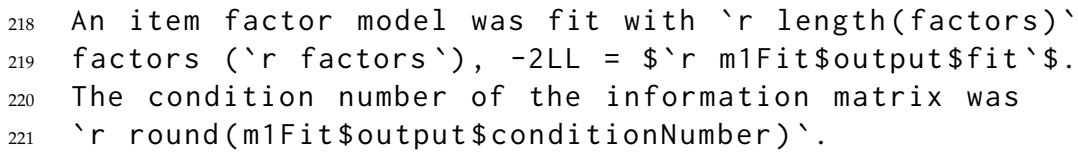

The boilerplate renders as, "An item factor model was fit with 1 factors (math), $-2 \mathrm{LL}=2767.48$. The condition number of the information matrix was 85.07." Since we have already seen much of the code to generate model diagnostics, we omit it here.

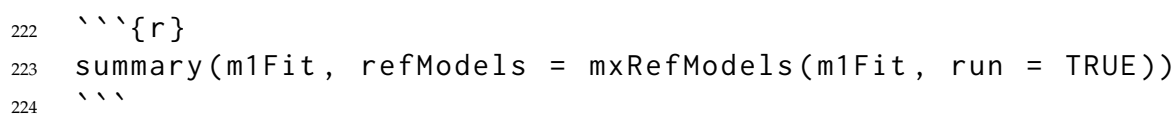


Summary of itemModel

free parameters:

name matrix row

1 itemModel item $[1,1]$ item math

2 eq1 item p3

3 itemModel.item[4,1] item p4

4 eq2 item p5

5 itemModel.item[6,1] item p6

col Estimate Std.Error

6 itemModel.item $[1,2]$

Match

$\begin{array}{lll}\text { Match } & 0.82 & 0.37\end{array}$

$\begin{array}{lll}\text { Match } & -1.18 & 0.27\end{array}$

$\begin{array}{lll}\text { Match } & 0.50 \quad 0.19\end{array}$

7 itemModel.item[4,2] item p4 Identify $-0.25 \quad 0.36$

8 itemModel.item[6,2] item p6 Identify $-1.40 \quad 0.21$

observed statistics: 15

estimated parameters: 8

degrees of freedom: 7

fit value ( $-2 \operatorname{lnL}$ units ): 2767

saturated fit value ( $-2 \operatorname{lnL}$ units ): 2758

number of observations: 592

chi-square: $\mathrm{x} 2(\mathrm{df}=7)=9.2, \quad \mathrm{p}=0.24$

Information Criteria:

| df Penalty | Parameters Penalty | Sample-Size Adjusted

AIC: $\quad 2753 \quad 2783 \quad$ NA

BIC: $\quad 2723 \quad 2819 \quad 2793$

CFI: 0.98

RMSEA: $0.023 \quad[95 \%$ CI $(0,0.064)]$

Prob(RMSEA $<=0.05): 0.88$

timestamp: 2016-02-24 10:15:07

Wall clock time (HH:MM:SS.hh): 00:00:04.55

OpenMx version number: 2.3.1.254

Need help? See help(mxSummary)

Although the outcomes are not strictly ordered for Identify in the item outcome map (Figure 21), other measures of model fit look reasonable. The sum-score item fit tests are not statistically significant at the 0.01 level (Table 3). This indicates good item-level fit. Since we requested a saturated and independence model (mxRefModels; line 223), CFI (Comparative Fit

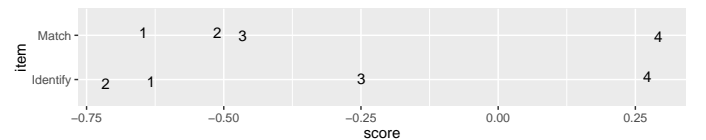

Figure 21: Item outcome by average latent score. Index), TLI (Tucker Lewis Index), and RMSEA (Root Mean Square Error of Approximation) are available in the OpenMx summary and suggest adequate model fit. These relative indices of fit are appropriate for these data because there are observations for all possible response patterns. However, be forewarned that as the multinomial table becomes more sparse, these indices become inaccurate. For sparse data, a more accurate assessment of model fit is available using other methods (Bartholomew and Tzamourani, 1999; Cai and Hansen, 2013).

\section{Rasch diagnostics}

A Rasch model is obtained when all slope parameters are constrained to be equal and the variance is fixed to 1.0, or equivalently, all slopes are fixed to 1.0 with free variance (Rasch, 1960/1993). If your interest is Rasch models with a single latent factor then you can take advantage of Rasch residual-based fit statistics. Infit and outfit are available from rpf.1dim. fit.

\section{Item factor analysis}

A common problem is that we do not know how many latent factors to employ to most accurately model our data. Fortunately, there is a method item factor analysis (Bock et al., 1988) analogous to factor analysis of continuous indicators (Lovie and Lovie, 1996). We will employ the likelihood ratio test for inference. The likelihood ratio test is asymptotically consistent for sparse multinomial 


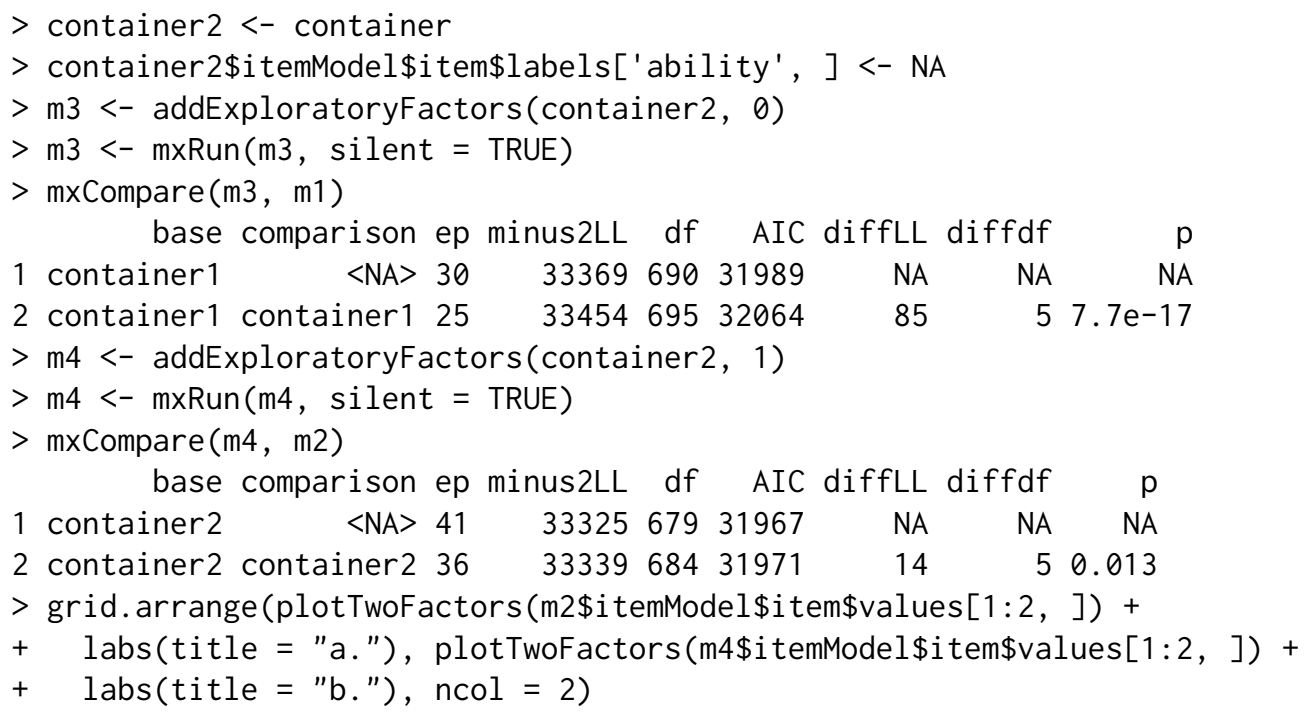

a.

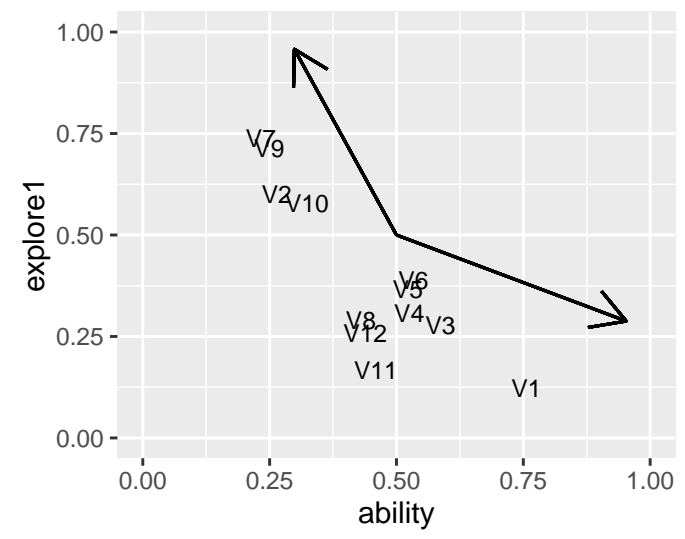

b.

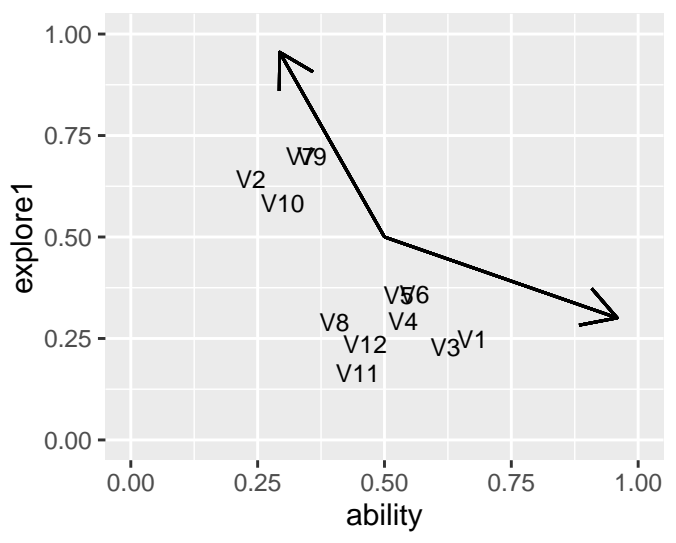

Figure 22: Factor loadings for items with (a) and without (b) the slope constraint. The code for plotTwoFactors is given in the Appendix.

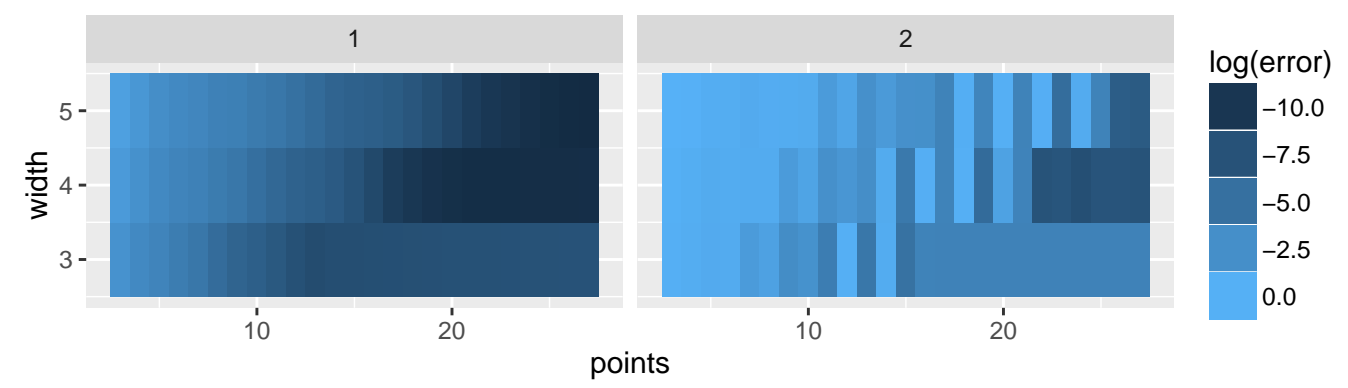

Figure 23: Log Euclidean distance $\left(l^{2}\right.$-norm) of error by quadrature width and number of points for 1 factor (left) and 2 factors (right). A wider width is important to accommodate data that conform less closely to a normal distribution. Even with clean simulated data, a width of 3 is too narrow and interferes with accuracy (both panes). In the 1 factor case (left), at least 21 points are required for high accuracy. For 2 factors (right), at least 23 points are required for a width of 4 and 27 points for a width of 5 . The bright strips at even numbers of point $(12,14,16$, etc) indicate that an odd number of points obtain somewhat better accuracy than even numbers of points.

distributions (Haberman, 1977). However, in finite samples, we should not expect that the null distribution is well calibrated. In brief, the $p$-values should not be taken too seriously.

$>\mathrm{m} 1<-$ addExploratoryFactors(container, 0 )
$>\mathrm{m} 1<-$ mxRun(m1, silent $=$ TRUE)
$>\mathrm{m} 2<-$ addExploratoryFactors(container, 1) 


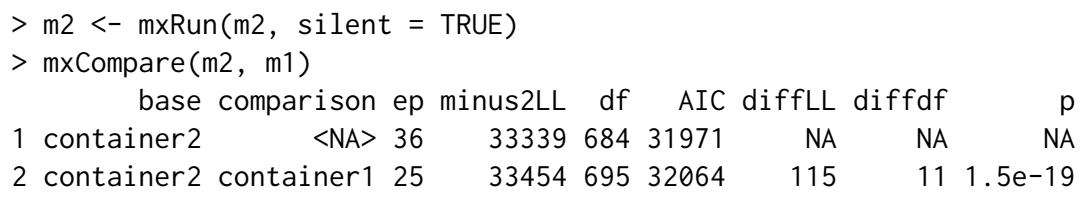

Here we find that there is reasonably good support in favor of a two factor solution. However, the slope of items 7-12 are constrained equal. Maybe this constraint was a mistake. It is possible that these items are well modeled by a single factor when all the slopes are freed. We cannot directly compare m2 against a single factor model without the slope constraint because these models are not nested. However, we can make a number of similar comparisons.

We find that there is a dramatic improvement in fit whether we relax the constraint on items 7-12 or we add another factor. Without knowing more about how the data were collected, parsimony favors a single factor model without constraints on the slopes. We can further check this idea by comparison of two factor models with and without the slope constraint (Figure 22).

A $p$-value of 0.013 is statistically significant at the customary 0.05 level, but we regard this as nonsignificant in comparison to the other $p$-values that are less than $10^{-16}$. We conclude that there is no difference between these models. For two factor models, it can be helpful to plot item factor loadings. A varimax rotation eliminates rotational indeterminacy. Promax axes are helpful to illustrate the rough directions of variability (Bock et al., 1988, p. 265). In both plots, the promax axes are separated by an angle close to $\pi$ radians, suggesting a single latent factor. The slight differences between plots (a) and (b) are probably due to overfitting. More precise $p$-values could be obtained using Monte Carlo techniques.

\section{Repercussions of the use of numerical quadrature for integration}

Recall that the optimization algorithm uses equal interval quadrature to evaluate the integral in Equation 2. It is important to understand how the quadrature grid influences model optimization accuracy and time. Let $Q$ be the number of quadrature points per dimension and $Q_{\text {width }}$ be the one-sided width of the quadrature for one dimension. Points $X_{q}$ are arranged as

$$
X_{q}=Q_{\text {width }}\left(1-\frac{2 q}{Q-1}\right) \text { for } q \in\{0, \ldots, Q-1\} .
$$

Generalization to more dimensions is accomplished by replication of the same 1 dimensional grid along each dimension. For example, a two factor model with 31 points per dimension involve $31^{2}=961$ grid points. Hence, optimization time is exponential in the number of general factors.

Figure 23 exhibits a simulation study of the influence of quadrature on model accuracy. All comparisons are against a 41 point quadrature of width 5.0. Before computing the Euclidean distance $\left(l^{2}\right.$-norm), the slope matrix was converted into factor loadings,

$$
\frac{\text { slope }}{\left[1+\operatorname{rowSums}\left(\text { slope }^{2}\right)\right]^{\frac{1}{2}}} \text {. }
$$

For two factor models, a varimax rotation was applied to eliminate rotational indeterminacy. The $l^{2}$-norm was applied to the resulting slope entries (ignoring intercepts). Each grid area in Figure 23 represents the average of 5 trials with different random starting values.

Item factor analysis with more than two factors requires patience and expertise. Model optimization time becomes an uncomfortable hindrance to experimentation. An optimization algorithm better suited to many latent factors, such as the Metropolis-Hastings Robbins-Monro algorithm (Cai, 2010b), is not yet available in OpenMx. The model builder offers as many as five factors because additional factors do not always increase estimation time. Suppose all items load on a general factor. In the special case that each item loads on at most one additional factor, many additional factors will not increase estimation time. One important use for this kind of factor structure is to account for local dependence (DeMars, 2006). For example, a reading comprehension test might have 3-4 items that relate to a single passage. The items within each passage will likely exhibit local dependence. One way to account for this kind of test structure is to add passage specific latent factors. Since the passages are disjoint, all of the passage specific factors will count as a single factor with respect to estimation time (Cai, 2010a). 


\section{Discussion}

We gave detailed instructions on how to set up IFA models for analysis of both dichotomous and polytomous data using the model builder app. We hope this will ease the learning curve for the construction of IFA models in OpenMx. The model builder app offers limited flexibility by design to reduce the number of options for novice users. For example, there is no facility for construction of multiple group models. This may be construed as a disadvantage, but we argue that keeping the app as simple as possible is important for newcomers to IFA. Learning OpenMx can be a daunting prospect. OpenMx, rpf, and ifaTools are free software. The source code is available for everybody to view, modify, and use. If you find this software useful, we hope you will cite us in your publications.

\section{Appendix}

Factors are plotted in a coordinate system determined by a varimax rotation (line 2). Promax axes are superimposed (line 9).

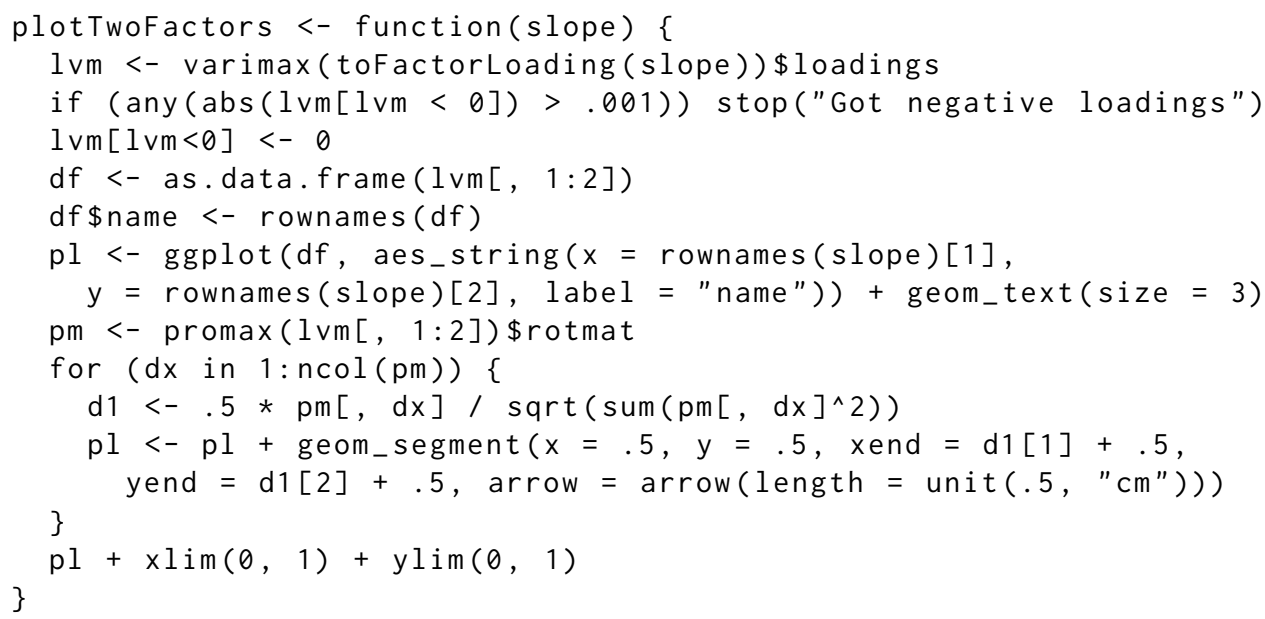

\section{Bibliography}

A. Agresti. Analysis of Categorical Data. Wiley, New York, 1990. [p192]

J. Allaire, J. McPherson, Y. Xie, H. Wickham, J. Cheng, and J. Allen. Rmarkdown: Dynamic Documents for $R$, 2014. URL http://rmarkdown. rstudio.com. R package version 0.3.8. [p182]

F. B. Baker and S. H. Kim. Item Response Theory: Parameter Estimation Techniques. CRC Press, 2nd edition, 2004. [p188]

D. J. Bartholomew and P. Tzamourani. The goodness of fit of latent trait models in attitude measurement. Sociological Methods E Research, 27(4):525-546, 1999. [p198]

A. Birnbaum. Some latent trait models and their use in inferring an examinee's ability. In F. M. Lord and M. R. Novick, editors, Statistical Theories of Mental Test Scores, pages 397-479. Addison-Wesley, Reading, MA, 1968. [p183, 184, 188]

R. D. Bock and M. Aitkin. Marginal maximum likelihood estimation of item parameters: Application of an EM algorithm. Psychometrika, 46:443-459, 1981. [p182]

R. D. Bock, R. Gibbons, and E. Muraki. Full-information item factor analysis. Applied Psychological Measurement, 12(3):261-280, 1988. [p198, 200]

L. Cai. A two-tier full-information item factor analysis model with applications. Psychometrika, 75(4): 581-612, 2010a. [p200]

L. Cai. High-dimensional exploratory item factor analysis by a Metropolis-Hastings Robbins-Monro algorithm. Psychometrika, 75(1):33-57, 2010b. [p182, 183, 200]

L. Cai and M. Hansen. Limited-information goodness-of-fit testing of hierarchical item factor models. British Journal of Mathematical and Statistical Psychology, 66(2):245-276, 2013. [p198] 
L. Cai, J. S. Yang, and M. Hansen. Generalized full-information item bifactor analysis. Psychological Methods, 16(3):221-248, 2011. [p189]

W.-H. Chen and D. Thissen. Local dependence indexes for item pairs using Item Response Theory. Journal of Educational and Behavioral Statistics, 22(3):265-289, 1997. [p192]

C. E. DeMars. Application of the bi-factor multidimensional Item Response Theory model to testletbased tests. Journal of Educational Measurement, 43(2):145-168, 2006. [p200]

A. Gelman. Objections to Bayesian statistics. Bayesian Analysis, 3(3):445-449, 2008. [p188]

S. J. Haberman. Log-linear models and frequency tables with small expected cell counts. The Annals of Statistics, 5(6):1148-1169, 11 1977. doi: 10.1214/aos/1176344001. [p199]

T. Kang and T. T. Chen. Performance of the generalized S- $X^{2}$ item fit index for polytomous IRT models. Journal of Educational Measurement, 45(4):391-406, 2008. [p193]

D. E. Knuth. Literate programming. The Computer Journal, 27(2):97-111, 1984. [p182]

Z. Li and L. Cai. Summed score likelihood based indices for testing latent variable distribution fit in item response theory. In Annual International Meeting of the Psychometric Society, Lincoln, NE, 2012. [p192]

E. Loken and K. L. Rulison. Estimation of a four-parameter item response theory model. British Journal of Mathematical and Statistical Psychology, 63(3):509-525, 2010. [p183, 184, 188]

P. Lovie and A. D. Lovie. Charles Edward Spearman, F.R.S. (1863-1945). Notes and Records of the Royal Society, 50(1):75-88, 1996. doi: 10.1098/rsnr.1996.0007. [p198]

D. Magis. A note on the item information function of the four-parameter logistic model. Applied Psychological Measurement, 37(4):304-315, 2013. [p188]

M. C. Neale, M. D. Hunter, J. N. Pritikin, M. Zahery, T. R. Brick, R. Kirkpatrick, R. Estabrook, T. C. Bates, H. Maes, and S. M. Boker. OpenMx 2.0: Extended structural equation and statistical modeling. Psychometrika, in press. doi: 10.1007/s11336-014-9435-8. [p182]

B. Nosek, G. Alter, G. Banks, D. Borsboom, S. Bowman, S. Breckler, S. Buck, C. Chambers, G. Chin, G. Christensen, et al. Promoting an open research culture. Science, 348(6242):1422-1425, 2015. [p182]

D. Oakes. Direct calculation of the information matrix via the EM algorithm. Journal of the Royal Statistical Society B (Statistical Methodology), 61(2):479-482, 1999. [p189]

M. Orlando and D. Thissen. Likelihood-based item-fit indices for dichotomous Item Response Theory models. Applied Psychological Measurement, 24(1):50-64, 2000. [p193]

R. D. Peng. Reproducible research in computational science. Science, 334(6060):1226, 2011. [p182]

J. N. Pritikin. ifaTools: Toolkit for Item Factor Analysis with OpenMx, 2015a. URL https://CRAN. Rproject. org/package=ifaTools. R package version 0.8. [p186]

J. N. Pritikin. rpf: Response Probability Functions, 2015b. URL https://CRAN. R-project.org/package= rpf. R package version 0.51. [p191]

J. N. Pritikin, M. D. Hunter, and S. M. Boker. Modular open-source software for Item Factor Analysis. Educational and Psychological Measurement, 75(3):458-474, 2015. doi: 10.1177/0013164414554615. [p182]

G. Rasch. Probabilistic Models for Some Intelligence and Attainment Tests. MESA Press, 1960/1993. [p198]

RStudio and Inc. shiny: Web Application Framework for R, 2014. URL http://CRAN. R-project.org/ package=shiny. R package version 0.10.2.1. [p182]

F. Samejima. Estimation of latent ability using a response pattern of graded scores. Psychometrika Monograph Supplement, 34(4):100, 1969. [p183]

D. Thissen and L. Steinberg. Data analysis using item response theory. Psychological Bulletin, 104(3): 385-395, 1988. [p195]

D. Thissen, L. Steinberg, and J. A. Mooney. Trace lines for testlets: A use of multiple-categoricalresponse models. Journal of Educational Measurement, 26(3):247-260, 1989. [p187] 
D. Thissen, L. Cai, and R. D. Bock. The Nominal Categories Item Response Model, pages 43-75. Routledge, 2010. [p183, 184, 189, 195]

W. M. Yen. Scaling performance assessments: Strategies for managing local item dependence. Journal of Educational Measurement, 30(3):187-213, 1993. [p192]

Joshua N. Pritikin

Department Psychology

University of Virginia

Charlottesville, VA 22904 USA

jpritikin@virginia.edu

Karen M. Schmidt

Department Psychology

University of Virginia

Charlottesville, VA 22904 USA

kschmidt@virginia.edu 